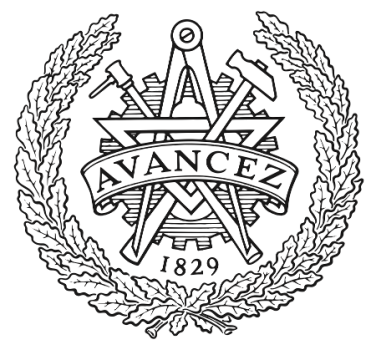

CHALMERS

UNIVERSITY OF TECHNOLOGY

\title{
Two-scale finite element modelling of reinforced concrete structures: Effective response and subscale fracture development
}

Downloaded from: https://research.chalmers.se, 2023-04-26 14:19 UTC

Citation for the original published paper (version of record):

Sciegaj, A., Larsson, F., Lundgren, K. et al (2018). Two-scale finite element modelling of reinforced concrete structures: Effective response and subscale fracture development. International Journal for Numerical Methods in Engineering, 114(10): 1074-1102. http://dx.doi.org/10.1002/nme.5776

N.B. When citing this work, cite the original published paper. 
This is the accepted version of the following article:

Sciegaj A, Larsson F, Lundgren K, Nilenius F, Runesson K. Two-scale finite element modelling of reinforced concrete structures: Effective response and subscale fracture development. International Journal for Numerical Methods in Engineering. 2018; 114(10): 1074-1102

which has been published in final form at https://doi.org/ 10.1002/nme. 5776 .

This article may be used for non-commercial purposes in accordance with the Wiley Self-Archiving Policy https: //authorservices.wiley.com/author-resources /

Journal-Authors/licensing-open-access/open-access/ self-archiving.html

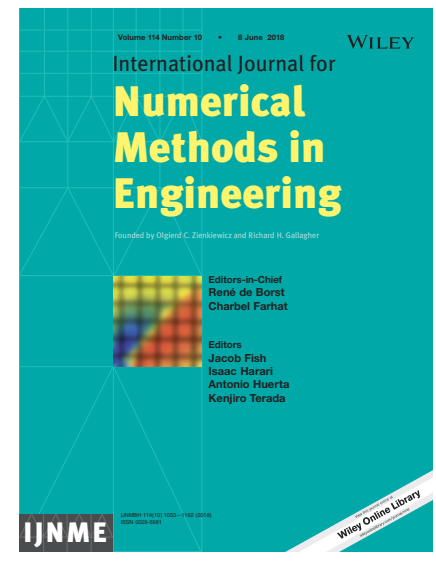




\title{
Two-scale finite element modelling of reinforced concrete structures: effective response and subscale fracture development
}

\author{
A. Sciegaj ${ }^{1,2}$, F. Larsson ${ }^{2}$, K. Lundgren ${ }^{1}$, F. Nilenius ${ }^{1}$, and K. Runesson ${ }^{2}$ \\ ${ }^{1}$ Department of Architecture and Civil Engineering \\ ${ }^{2}$ Department of Industrial and Materials Science \\ Chalmers University of Technology, Gothenburg, Sweden
}

\begin{abstract}
A two-scale model is derived from a fully resolved model, where the response of concrete, steel reinforcement, as well as bond between them, are considered. The pertinent "effective" large-scale problem is derived from selective homogenisation in terms of the equilibrium of reinforced concrete considered as a single-phase solid. Variational formulations of the Representative Volume Element (RVE) problem are established in terms of the subscale displacement fields for the plain concrete continuum and the reinforcement bars. Dirichlet and Neumann boundary conditions are imposed on the concrete (pertaining to uniform boundary displacement and constant boundary traction, respectively), and on the reinforcement bars (pertaining to prescribed boundary displacement and vanishing sectional forces, respectively). Different RVE sizes and combinations of boundary conditions were used in $\mathrm{FE}^{2}$ analyses of a deep beam subjected to four point bending. Results were compared with those of full resolution (single-scale). The most reliable response was obtained for the case of Dirichlet-Dirichlet (DD) boundary conditions, with a good match between the models in terms of the deformed shape, force-deflection relation and average strain. Even though the maximum crack widths were underestimated, the DD combination provided an approximate upper bound on the structural stiffness.
\end{abstract}

Keywords: reinforced concrete; computational homogenisation; multiscale; RVE; bond; cracking

\section{Introduction}

Crack growth in reinforced concrete is of practical importance; it does not only influence the shortterm response of the structure, but it also affects the durability in the sense that cracks facilitate the ingress of harmful substances like chlorides, which in turn can cause corrosion of the reinforcement. In the analysis of crack growth it is also necessary to consider the bond between the reinforcement bars and the surrounding concrete, cf. [1, 2]. According to the ACI Committee [3], the bond mechanism results from chemical adhesion, friction and mechanical interlocking between the ribs of the reinforcement bars and the concrete. The resistance from chemical adhesion is small and is lost almost immediately when slipping between steel and concrete begins. In the course of the ensuing bond action, stresses between ribs and concrete are generated. More specifically, the traction is conveniently decomposed into shear and normal components, denoted bond and splitting stress, respectively. A common way to model the bond between concrete and reinforcement is to relate the bond stress to the slip (difference in tangential displacement of steel and concrete along a bar), see e.g. Model Code 2010 [4] and Domínguez [5].

Since concrete has a heterogeneous microstructure, the prediction of its mechanical response requires models that account for the complicated composite behaviour. One possibility of modelling 
reinforced concrete is to use a multiscale modelling strategy, more specifically the $\mathrm{FE}^{2}$ approach, which takes the coupling between different geometrical scales into account. For many applications, the separation of length scales can be assumed, and the macroscale response is obtained through computational homogenisation of the response of the subscale problem on a Representative Volume Element (RVE), cf. [6, 7] . Although the concurrent solution of a problem on two scales are computationally demanding, the approach is well suited for parallel computation in view of the fact that each RVE problem can be solved separately.

Multiscale approaches have been employed for plain concrete in a number of studies, cf. [8, 9]. For example, cement paste, aggregates and interfacial transition zones (ITZ) have been analysed in a multiscale manner in $[10,11,12]$. Strain localisation has also been the object of interest for a number of studies. For example, a crack modelling framework based on computational homogenisation, where a microscale localisation band is aggregated to a macroscale discontinuous crack, has been presented for plain concrete by Nguyen et al. [13]. A computational homogenisation-localisation nested scheme bridging the microscale damage and macroscopic failure has been developed by Coenen et al. [14]. Even though only quasi-static loading was considered in the mentioned works, multiscale ratedependent crack models have also been studied. Karamnejad et al. [15] presented a multiscale approach to modelling cracking in heterogeneous quasi-brittle materials subjected to dynamic load.

However, reinforced concrete has not been investigated to the same extent. Examples of how to link different length scales when modelling reinforced concrete involve the studies conducted by Le et al. [16] and Sun and $\operatorname{Li}[17,18,19]$. In [18], an adaptive multiscale method, employing mesoscale image-based modelling, has been developed to study the response and damage evolution in reinforced concrete. However, the reinforcement was not included explicitly in the analysed RVEs and the approach was not based on the separation of length scales; instead, a spatially concurrent multiscale formulation was used. The use of concurrent multiscale methods, which is similar to submodelling, typically requires the full resolution of damaged parts of the structure. For distributed cracking (e.g. in the presence of reinforcement grids) this would require resolving the underlying sub-structure almost everywhere, making it computationally far too expensive. Therefore, there is a need for a multiscale modelling framework for reinforced concrete based on computational homogenisation.

In this paper, a novel two-scale procedure for the analysis of reinforced concrete structures subjected to quasi-static loading is devised and studied in terms of its ability to predict (i) the overall response (stress-strain) and (ii) development of subscale fracture. The variationally consistent homogenisation approach proposed by Larsson et al. [20] is used to derive the two-scale formulation. The relevant equilibrium equations are established for a subscale RVE including concrete, steel reinforcement, and the interaction (bond) mechanism. First-order homogenisation is used to obtain the macroscopic response, thus creating an "apparent" constitutive model of the effective material. It is noteworthy, that the homogenisation is selective in the sense that it is only the concrete displacement that possesses an independent macroscopic component.

The paper is organised as follows: The fully resolved problem is established in Section 2. The expression "fully resolved" is used to emphasise the strong subscale heterogeneity of the material, i.e. concrete, reinforcing bars and interfaces between these can be distinguished and analysed. In Section 3 the assumption of separation of scales and variationally consistent computational homogenisation are used to derive the corresponding two-scale formulation. The pertinent large-scale and subscale problems are established and described. The nested finite element algorithm for solving the two-scale problem is presented in Section 4. In Section 5, the effective response of the reinforced concrete RVE is studied in detail. Section 6 comprises the application of the developed two-scale model to a deep reinforced concrete beam subjected to four point bending. Computational results of the two-scale analyses are compared with those of a conventional single-scale analysis. The paper 
is concluded with Section 7, which contains some final remarks and specifies issues to be addressed in future studies.

\section{Fully resolved problem}

In this section, the variational format of the governing field equations for a boundary value problem that represents quasistatic loading of a a reinforced concrete structure, is derived. Since modelling of large reinforced concrete structures is in focus, it is assumed in the following that plain concrete can be treated as a two-dimensional continuum, and that the reinforcement has beam-like behaviour, i.e. that it can sustain both bending moments and normal force. For other types of reinforced concrete structures, especially for those modelled in three dimensions, it could be worthwhile to include heterogeneities (aggregates, cement paste, interfacial transition zone and reinforcement) while modelling concrete. However, such extensions are left outside the scope of the present study.

\subsection{Strong format}

We consider a reinforced concrete structure, that is simplified as a two-dimensional model, e.g. a relatively thin member in a state of plane stress. A symbolic representation is given in Figure 1, where the grid represents intersecting reinforcement bars. The problem domain $\Omega=\Omega_{\mathrm{c}} \cup \Gamma_{\text {int }}$ comprises the concrete $\left(\Omega_{\mathrm{c}}\right)$ and reinforcement $\left(\Gamma_{\mathrm{int}}\right)$ parts. For each part of $\Gamma_{\text {int }}$ it is possible to define unit vectors $\boldsymbol{e}_{1}$ and $\boldsymbol{e}_{\perp}$, in the longitudinal and transverse direction, respectively. The outer boundary $\Gamma_{\text {ext }}:=\partial \Omega=\Gamma_{\mathrm{u}} \cup \Gamma_{\mathrm{t}}$ is split into Dirichlet $\left(\Gamma_{\mathrm{u}}\right)$ and Neumann $\left(\Gamma_{\mathrm{t}}\right)$ parts with prescribed displacements and traction, respectively. Since the reinforcement can be considered as idealised one-dimensional segments, the boundary of $\Omega_{\mathrm{c}}$ can be expressed as $\partial \Omega_{\mathrm{c}}=\Gamma_{\text {ext }} \cup \Gamma_{\text {int }}$. Moreover, it is assumed that the reinforcement does not cross the external boundary $\Gamma_{\text {ext }}$, i.e. that each bar ends within the structure. Hence, $\Gamma_{\text {int }} \cap \Gamma_{\text {ext }}=\varnothing$.

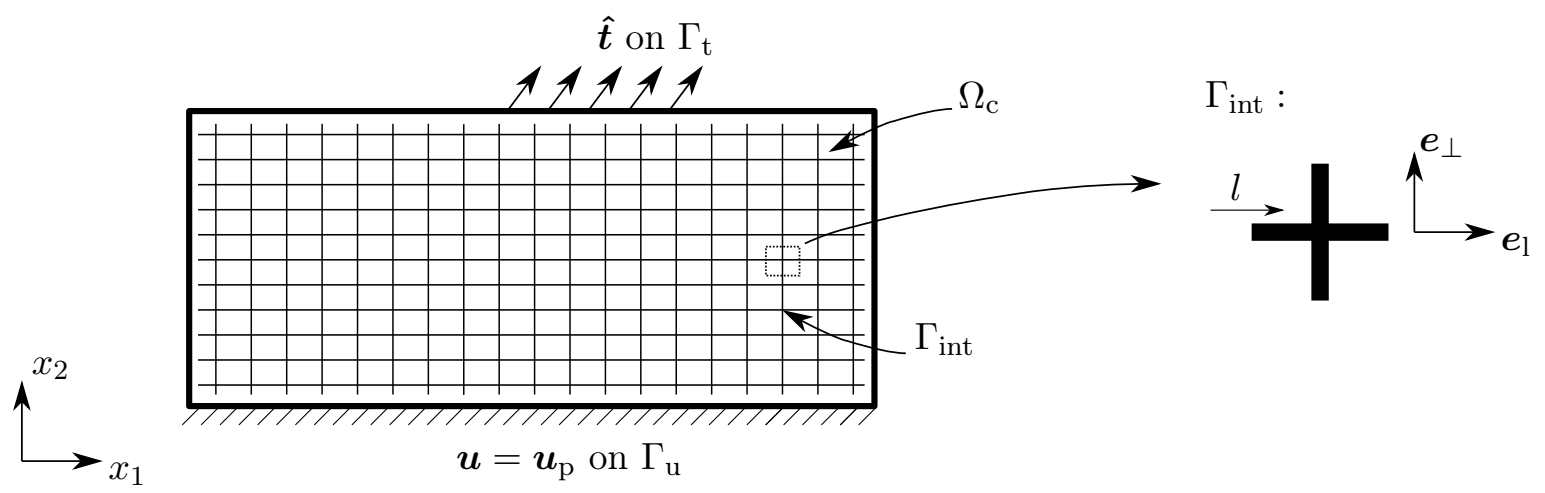

Figure 1: A two-dimensional reinforced concrete structure.

For the 2D problem, displacement fields that are pertinent to concrete and steel are denoted $\boldsymbol{u}_{\mathrm{c}}(\boldsymbol{x})$ : $\Omega \rightarrow \mathbb{R}^{2}$ and $\boldsymbol{u}_{\mathrm{s}}(l): \Gamma_{\text {int }} \rightarrow \mathbb{R}^{2}$ respectively. It is assumed that $\boldsymbol{u}_{\mathrm{c}}$ is continuous across $\Gamma_{\text {int. }}$. Along $\Gamma_{\text {int }}$, it is possible to split $\boldsymbol{u}_{\mathrm{s}}$ and $\boldsymbol{u}_{\mathrm{c}}$ into components that are parallel and perpendicular to $\Gamma_{\text {int }}$, i.e.

$$
\begin{aligned}
& \boldsymbol{u}_{\mathrm{s}}=u_{\mathrm{s}, 1} \boldsymbol{e}_{\mathrm{l}}+u_{\mathrm{s}, \perp} \boldsymbol{e}_{\perp}, \\
& \boldsymbol{u}_{\mathrm{c}}=u_{\mathrm{c}, \mathrm{l}} \boldsymbol{e}_{\mathrm{l}}+u_{\mathrm{c}, \perp} \boldsymbol{e}_{\perp} .
\end{aligned}
$$


The equilibrium equation for the concrete phase takes the form

$$
\begin{gathered}
-\left(t_{\mathrm{c}} \boldsymbol{\sigma}_{\mathrm{c}}\right) \cdot \boldsymbol{\nabla}=t_{\mathrm{c}} \boldsymbol{b} \quad \text { in } \Omega_{\mathrm{c}}, \\
\boldsymbol{u}=\boldsymbol{u}_{\mathrm{p}} \quad \text { on } \Gamma_{\mathrm{u}}, \quad \boldsymbol{t}:=\boldsymbol{\sigma}_{\mathrm{c}} \cdot \boldsymbol{n}=\hat{\boldsymbol{t}} \quad \text { on } \Gamma_{\mathrm{t}},
\end{gathered}
$$

where $t_{\mathrm{c}}$ is the thickness of the structure, $\boldsymbol{b}$ is the body force and $\boldsymbol{\sigma}_{\mathrm{c}}$ is the stress in the concrete phase. Prescribed displacement $\boldsymbol{u}_{\mathrm{p}}$ and traction $\hat{\boldsymbol{t}}$ are acting on the Dirichlet and Neumann parts of the boundary, respectively.

The reinforcing bars occupying the region $\Gamma_{\text {int }}$ can be analysed separately as truss/bar or beam elements to account for longitudinal and transverse load effects, respectively. To begin with, the longitudinal equilibrium is established, where the normal force $N_{\mathrm{s}}$ is linked to the bond stresses (denoted $t_{\Gamma}$ ) that are distributed around the circumference $S_{\mathrm{s}}$ of the bar:

$$
\begin{aligned}
-\frac{\partial N_{\mathrm{s}}}{\partial l}+S_{\mathrm{s}} t_{\Gamma}=0 & \text { in } \Gamma_{\text {int }}, \\
N_{\mathrm{s}}=0 & \text { on } \partial \Gamma_{\text {int }} .
\end{aligned}
$$

The transverse action of the reinforcement is modelled using Euler-Bernoulli beam theory. In this case, the governing equation linking the shear force $T_{\mathrm{S}}=\partial M_{\mathrm{S}} / \partial l$ and bending moment $M_{\mathrm{S}}$ to transversely distributed load $\lambda$ is

$$
\begin{array}{ll}
-\frac{\partial^{2} M_{\mathrm{s}}}{\partial l^{2}}+\lambda=0 & \text { in } \Gamma_{\mathrm{int}}, \\
T_{\mathrm{s}}=0, M_{\mathrm{s}}=0 & \text { on } \partial \Gamma_{\mathrm{int}} .
\end{array}
$$

Lastly, it is assumed that there is no relative motion between steel and concrete in the transverse direction, i.e. the interface constraint has the form:

$$
u_{\mathrm{s}, \perp}-u_{\mathrm{c}, \perp}=0
$$

In view of the constraint (6), the transverse load $\lambda$ can be interpreted as a Lagrange multiplier. Next, we consider the steel/concrete interface and formulate the tangential equilibrium condition, cf. Figure 2. The left and right parts of the concrete continuum have normal vectors $\boldsymbol{n}^{-}$and $\boldsymbol{n}^{+}$, while the stress tensors are denoted $\sigma_{\mathrm{c}}^{-}$and $\boldsymbol{\sigma}_{\mathrm{c}}^{+}$, respectively. The reinforcing bar subjected to bond stresses and transverse load has been protruded out of the concrete for clarity, and the remaining husk inside the concrete part is subjected to the same forces with opposite signs. It will be assumed that the reinforcement carries the entire load over $\Gamma_{\text {int }}$, whereby the following equilibrium equation can be established for the concrete part along $\Gamma_{\text {int }}$ :

$$
\left[t_{\mathrm{c}}^{-} \boldsymbol{\sigma}_{\mathrm{c}}^{-}\right] \cdot \boldsymbol{e}_{\perp}-\left[t_{\mathrm{c}}^{+} \boldsymbol{\sigma}_{\mathrm{c}}^{+}\right] \cdot \boldsymbol{e}_{\perp}+\lambda \boldsymbol{e}_{\perp}+S_{\mathrm{s}} t_{\Gamma} \boldsymbol{e}_{\mathrm{l}}=0 .
$$

Up to this point, no constitutive relations have been defined for the materials. To maintain generality, only implicit (algorithmic) definitions of the constitutive relations will be given. Hence, although evolving internal variables are considered, they are omitted in the abstract notation below. Thus, for the concrete we have a general relation between the stress and strain tensors, which can be written as

$$
\boldsymbol{\sigma}_{\mathrm{c}}=\boldsymbol{\sigma}_{\mathrm{c}}\left(\varepsilon_{\mathrm{c}}\left[\boldsymbol{u}_{\mathrm{c}}\right]\right)
$$




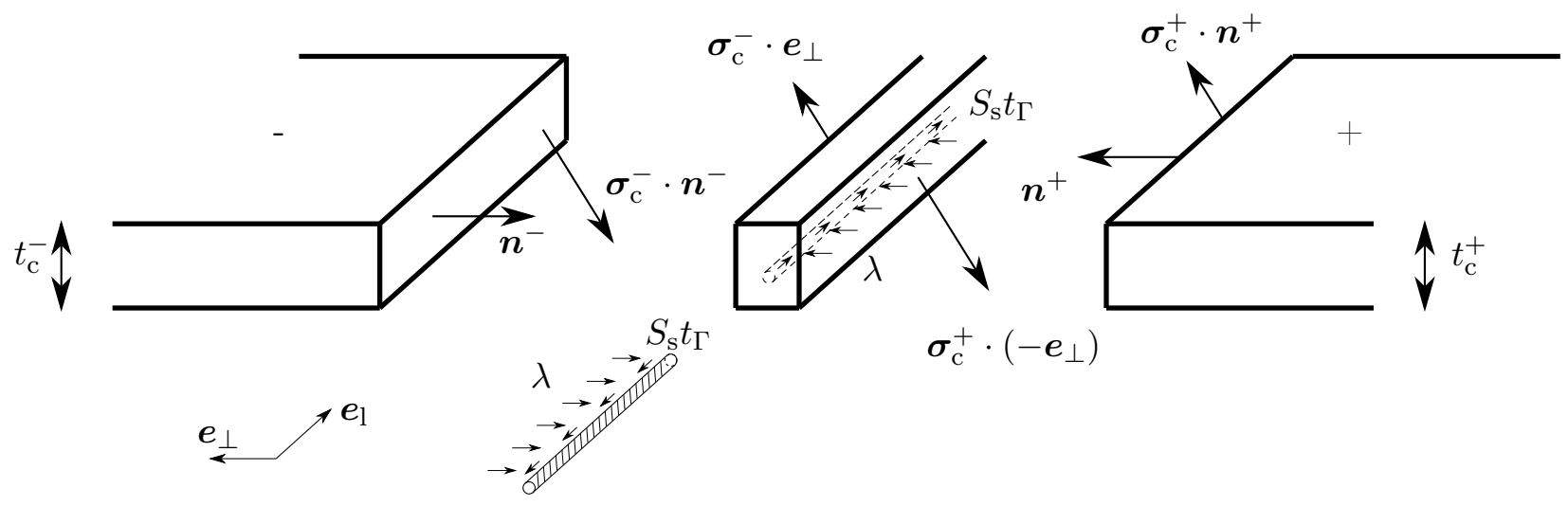

Figure 2: Steel/concrete interface with all the forces acting on it.

where $\varepsilon_{\mathrm{c}}=\left[\boldsymbol{u}_{\mathrm{c}} \otimes \nabla\right]^{\mathrm{sym}}$ denotes the strain, For the reinforcement, we consider the constitutive relation as the one linking internal forces to the displacement of the bar, i.e.

$$
\begin{aligned}
& N_{\mathrm{s}}=N_{\mathrm{s}}\left(\frac{\partial u_{\mathrm{s}, 1}}{\partial l}, \frac{\partial^{2} u_{\mathrm{s}, \perp}}{\partial l^{2}}\right), \\
& M_{\mathrm{s}}=M_{\mathrm{s}}\left(\frac{\partial u_{\mathrm{s}, 1}}{\partial l}, \frac{\partial^{2} u_{\mathrm{s}, \perp}}{\partial l^{2}}\right) .
\end{aligned}
$$

The remaining constitutive relation concerns the tangential bond stress $t_{\Gamma}$, which is assumed to depend on the longitudinal slip $u_{\mathrm{s}, 1}-u_{\mathrm{c}, \mathrm{l}}$. The bond-slip relation is defined as

$$
t_{\Gamma}=t_{\Gamma}\left(u_{\mathrm{s}, 1}-u_{\mathrm{c}, 1}\right) .
$$

\subsection{Variational format}

If the body forces are neglected, the weak format of (3) becomes

$$
\int_{\Omega_{\mathrm{c}}} t_{\mathrm{c}} \boldsymbol{\sigma}_{\mathrm{c}}:\left[\delta \boldsymbol{u}_{\mathrm{c}} \otimes \nabla\right] \mathrm{d} \Omega-\int_{\partial \Omega_{\mathrm{c}}}\left[t_{\mathrm{c}} \boldsymbol{\sigma}_{\mathrm{c}} \cdot \boldsymbol{n}\right] \cdot \delta \boldsymbol{u}_{\mathrm{c}} \mathrm{d} \Gamma=0,
$$

for a suitable choice of test function $\delta \boldsymbol{u}_{\mathrm{c}}$ (to be specified later). The boundary $\partial \Omega_{\mathrm{c}}$ can be split according to Figure 3. Since $\boldsymbol{n}^{+}=\boldsymbol{e}_{\perp}$ and $\boldsymbol{n}^{-}=-\boldsymbol{e}_{\perp}$, the boundary term becomes

$$
\int_{\partial \Omega_{\mathrm{c}}}\left[t_{\mathrm{c}} \boldsymbol{\sigma}_{\mathrm{c}} \cdot \boldsymbol{n}\right] \cdot \delta \boldsymbol{u}_{\mathrm{c}} \mathrm{d} \Gamma=\int_{\Gamma_{\mathrm{ext}}}\left[t_{\mathrm{c}} \boldsymbol{\sigma}_{\mathrm{c}} \cdot \boldsymbol{n}\right] \cdot \delta \boldsymbol{u}_{\mathrm{c}} \mathrm{d} \Gamma+\int_{\Gamma_{\mathrm{int}}}\left[\left[t_{\mathrm{c}}^{+} \boldsymbol{\sigma}_{\mathrm{c}}^{+}-t_{\mathrm{c}}^{-} \boldsymbol{\sigma}_{\mathrm{c}}^{-}\right] \cdot \boldsymbol{e}_{\perp}\right] \cdot \delta \boldsymbol{u}_{\mathrm{c}} \mathrm{d} \Gamma .
$$

With (7), we rewrite (13) as

$$
\int_{\partial \Omega_{\mathrm{c}}}\left[t_{\mathrm{c}} \boldsymbol{\sigma}_{\mathrm{c}} \cdot \boldsymbol{n}\right] \cdot \delta \boldsymbol{u}_{\mathrm{c}} \mathrm{d} \Gamma=\int_{\Gamma_{\mathrm{ext}}}\left[t_{\mathrm{c}} \boldsymbol{\sigma}_{\mathrm{c}} \cdot \boldsymbol{n}\right] \cdot \delta \boldsymbol{u}_{\mathrm{c}} \mathrm{d} \Gamma+\int_{\Gamma_{\mathrm{int}}} \lambda \boldsymbol{e}_{\perp} \cdot \delta \boldsymbol{u}_{\mathrm{c}} \mathrm{d} \Gamma+\int_{\Gamma_{\mathrm{int}}} S_{\mathrm{s}} t_{\Gamma} \boldsymbol{e}_{1} \cdot \delta \boldsymbol{u}_{\mathrm{c}} \mathrm{d} \Gamma
$$

Finally, combining (14) and (12), we obtain the weak form of the governing equation for the concrete continuum

$$
\int_{\Omega_{\mathrm{c}}} t_{\mathrm{c}} \boldsymbol{\sigma}_{\mathrm{c}}:\left[\delta \boldsymbol{u}_{\mathrm{c}} \otimes \nabla\right] \mathrm{d} \Omega-\int_{\Gamma_{\mathrm{int}}} \lambda \delta u_{\mathrm{c}, \perp} \mathrm{d} \Gamma-\int_{\Gamma_{\mathrm{int}}} S_{\mathrm{s}} t_{\Gamma} \delta u_{\mathrm{c}, \mathrm{l}} \mathrm{d} \Gamma=\int_{\Gamma_{\mathrm{ext}}}\left[t_{\mathrm{c}} \boldsymbol{\sigma}_{\mathrm{c}} \cdot \boldsymbol{n}\right] \cdot \delta \boldsymbol{u}_{\mathrm{c}} \mathrm{d} \Gamma .
$$




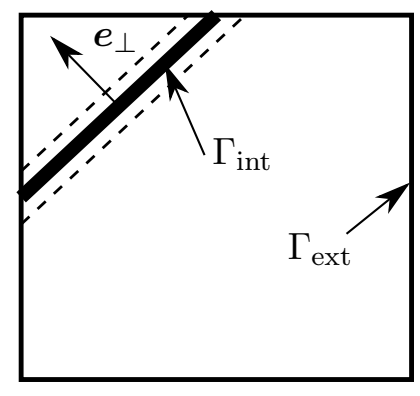

Figure 3: Constituents of the boundary $\partial \Omega_{\mathrm{c}}$.

Next, similar arguments are applied to (4) and (5). With the relevant boundary conditions, (4) is recast as:

$$
\int_{\Gamma_{\mathrm{int}}} N_{\mathrm{s}} \frac{\partial \delta u_{\mathrm{s}, 1}}{\partial l} \mathrm{~d} \Gamma+\int_{\Gamma_{\mathrm{int}}} S_{\mathrm{s}} t_{\Gamma} \delta u_{\mathrm{s}, 1} \mathrm{~d} \Gamma=0
$$

for a suitable choice of test function $\delta u_{\mathrm{s}, 1}$ (to be defined later). Likewise, (5) is recast as

$$
-\int_{\Gamma_{\mathrm{int}}} M_{\mathrm{s}} \frac{\partial^{2} \delta u_{\mathrm{s}, \perp}}{\partial l^{2}} \mathrm{~d} \Gamma+\int_{\Gamma_{\mathrm{int}}} \lambda \delta u_{\mathrm{s}, \perp} \mathrm{d} \Gamma=0
$$

with a suitable choice of test function $\delta u_{\mathrm{s}, \perp}$ (to be defined later). Finally, the interface constraint (6) can be formulated in the weak sense as

$$
\int_{\Gamma_{\mathrm{int}}}\left[u_{\mathrm{s}, \perp}-u_{\mathrm{c}, \perp}\right] \delta \lambda \mathrm{d} \Gamma=0
$$

for a suitable choice of test function $\delta \lambda$ (to be specified later). Recall that the constraint parameter $\lambda$ plays the role of a Lagrange multiplier.

The fully resolved quasi-static problem can now be defined in the variational form as follows: Find $\boldsymbol{u}_{\mathrm{c}}, u_{\mathrm{s}, 1}, u_{\mathrm{s}, \perp}, \lambda \in \mathbb{U}_{\mathrm{c}} \times \mathbb{U}_{\mathrm{s}, 1} \times \mathbb{U}_{\mathrm{s}, \perp} \times \mathbb{L}$ such that

$$
\begin{aligned}
a_{\mathrm{c}}\left(\boldsymbol{u}_{\mathrm{c}} ; \delta \boldsymbol{u}_{\mathrm{c}}\right)-b\left(u_{\mathrm{s}, 1}-\boldsymbol{e}_{\mathrm{l}} \cdot \boldsymbol{u}_{\mathrm{c}} ; \boldsymbol{e}_{\mathrm{l}} \cdot \delta \boldsymbol{u}_{\mathrm{c}}\right)-c\left(\lambda ; \boldsymbol{e}_{\perp} \cdot \delta \boldsymbol{u}_{\mathrm{c}}\right) & =l_{\mathrm{c}}\left(\delta \boldsymbol{u}_{\mathrm{c}}\right) & \forall \delta \boldsymbol{u}_{\mathrm{c}} \in \mathbb{U}_{\mathrm{c}}^{0}, \\
a_{\mathrm{l}}\left(u_{\mathrm{s}, 1}, u_{\mathrm{s}, \perp} ; \delta u_{\mathrm{s}, 1}\right)+b\left(u_{\mathrm{s}, 1}-\boldsymbol{e}_{\mathrm{l}} \cdot \boldsymbol{u}_{\mathrm{c}} ; \delta u_{\mathrm{s}, 1}\right) & =0 & \forall \delta u_{\mathrm{s}, 1} \in \mathbb{U}_{\mathrm{s}, 1}, \\
a_{\mathrm{b}}\left(u_{\mathrm{s}, 1}, u_{\mathrm{s}, \perp} ; \delta u_{\mathrm{s}, \perp}\right)+c\left(\lambda ; \delta u_{\mathrm{s}, \perp}\right) & =0 & \forall \delta u_{\mathrm{s}, \perp} \in \mathbb{U}_{\mathrm{s}, \perp}, \\
c\left(\delta \lambda ; u_{\mathrm{s}, \perp}-\boldsymbol{e}_{\perp} \cdot \boldsymbol{u}_{\mathrm{c}}\right) & =0 & \forall \delta \lambda \in \mathbb{L},
\end{aligned}
$$

for suitable trial sets $\mathbb{U}_{\mathrm{c}}, \mathbb{U}_{\mathrm{s}, 1}, \mathbb{U}_{\mathrm{s}, \perp}, \mathbb{L}$ :

$$
\begin{aligned}
\mathbb{U}_{\mathrm{c}} & =\left\{\boldsymbol{u}(\boldsymbol{x}): \Omega \mapsto \mathbb{R}^{2}, \int_{\Omega_{\mathrm{c}}} \boldsymbol{u}^{2}+[\boldsymbol{u} \otimes \boldsymbol{\nabla}]^{2} \mathrm{~d} \Omega<\infty, \boldsymbol{u}=\boldsymbol{u}_{\mathrm{p}} \text { on } \Gamma_{\mathrm{p}}\right\}, \\
\mathbb{U}_{\mathrm{s}, l} & =\left\{v(l): \Gamma_{\mathrm{int}} \mapsto \mathbb{R}, \int_{\Gamma_{\mathrm{int}}} v^{2}+\left(\frac{\partial v}{\partial l}\right)^{2} \mathrm{~d} \Gamma<\infty\right\}, \\
\mathbb{U}_{\mathrm{s}, \perp} & =\left\{v(l): \Gamma_{\mathrm{int}} \mapsto \mathbb{R}, \int_{\Gamma_{\mathrm{int}}} v^{2}+\left(\frac{\partial v}{\partial l}\right)^{2}+\left(\frac{\partial^{2} v}{\partial l^{2}}\right)^{2} \mathrm{~d} \Gamma<\infty\right\}, \\
\mathbb{L} & =\left\{\lambda(l): \Gamma_{\mathrm{int}} \mapsto \mathbb{R}, \int_{\Gamma_{\mathrm{int}}} \lambda^{2} \mathrm{~d} \Gamma<\infty\right\}
\end{aligned}
$$


and the test space $\mathbb{U}_{\mathrm{c}}^{0}$ :

$$
\mathbb{U}_{\mathrm{c}}^{0}=\left\{\boldsymbol{u}(\boldsymbol{x}): \Omega \mapsto \mathbb{R}^{2}, \int_{\Omega_{\mathrm{c}}} \boldsymbol{u}^{2}+[\boldsymbol{u} \otimes \nabla]^{2} \mathrm{~d} \Omega<\infty, \boldsymbol{u}=\mathbf{0} \text { on } \Gamma_{\mathrm{p}}\right\} .
$$

The coupling terms in the system (19)-(22) are defined as:

$$
\begin{aligned}
b(v ; w) & :=\int_{\Gamma_{\mathrm{int}}} S_{\mathrm{s}} t_{\Gamma}(v) w \mathrm{~d} \Gamma, \\
c(\lambda ; v) & :=\int_{\Gamma_{\mathrm{int}}} \lambda v \mathrm{~d} \Gamma .
\end{aligned}
$$

The following forms are introduced pertinent to:

(i) Concrete:

$$
\begin{aligned}
a_{\mathrm{c}}\left(\boldsymbol{u}_{\mathrm{c}} ; \delta \boldsymbol{u}_{\mathrm{c}}\right) & :=\int_{\Omega_{\mathrm{c}}} t_{\mathrm{c}} \boldsymbol{\sigma}_{\mathrm{c}}\left(\varepsilon\left[\boldsymbol{u}_{\mathrm{c}}\right]\right):\left[\delta \boldsymbol{u}_{\mathrm{c}} \otimes \nabla\right] \mathrm{d} \Omega, \\
l_{\mathrm{c}}\left(\delta \boldsymbol{u}_{\mathrm{c}}\right) & :=\int_{\Gamma_{\mathrm{ext}}} t_{\mathrm{c}} \hat{\boldsymbol{t}} \cdot \delta \boldsymbol{u}_{\mathrm{c}} \mathrm{d} \Gamma .
\end{aligned}
$$

(ii) Bar action of the rebars:

$$
a_{\mathrm{l}}\left(u_{\mathrm{s}, 1}, u_{\mathrm{s}, \perp} ; \delta u_{\mathrm{s}, 1}\right):=\int_{\Gamma_{\mathrm{int}}} N_{\mathrm{s}}\left(\frac{\partial u_{\mathrm{s}, 1}}{\partial l}, \frac{\partial^{2} u_{\mathrm{s}, \perp}}{\partial l^{2}}\right) \frac{\partial \delta u_{\mathrm{s}, 1}}{\partial l} \mathrm{~d} \Gamma .
$$

(iii) Beam action of the rebars:

$$
a_{\mathrm{b}}\left(u_{\mathrm{s}, 1}, u_{\mathrm{s}, \perp} ; \delta u_{\mathrm{s}, \perp}\right):=-\int_{\Gamma_{\mathrm{int}}} M_{\mathrm{s}}\left(\frac{\partial u_{\mathrm{s}, \mathrm{l}}}{\partial l}, \frac{\partial^{2} u_{\mathrm{s}, \perp}}{\partial l^{2}}\right) \frac{\partial^{2} \delta u_{\mathrm{s}, \perp}}{\partial l^{2}} \mathrm{~d} \Gamma .
$$

\section{Formulation of two-scale problem}

In multiscale modelling, the classical terms macroscale and microscale are most often used in the literature to distinguish between the level of detail with which the material is modelled. Since in this paper, reinforced concrete is treated as a continuum (the plain concrete) with embedded distinct reinforcement bars, the physical length scale of such a composite is clearly macroscopic. Moreover, as previously alluded to in Section 2, it would also be possible to consider the plain concrete as a heterogeneous material comprising aggregates, cement paste, and the interfacial transition zone (which physically corresponds to mesoscale). In order to better reflect the physical nature of the problem, the classically used terms macroscale and microscale are substituted, in this paper, with the terms large-scale and subscale. In the following, whenever the term macroscale is used, it pertains to the physical scale of the structure (large-scale).

\subsection{Preliminaries}

Solving for the fine-scale response directly from (19)-(22) is not always feasible for large reinforced concrete structures due to the required extensive computational effort. It is, therefore, expedient to divide the overall response into a large-scale and a subscale (fluctuation) part. The scale separation 
technique is thoroughly treated in [20, 21] and is referred to as Variationally Consistent Homogenisation. By use of the variational multiscale (VMS) ansatz, the unknown fields $\boldsymbol{u}_{\mathrm{c}}, u_{\mathrm{s}, 1}, u_{\mathrm{s}, \perp}$ can be separated into the macroscale (smooth) and subscale (fluctuating) parts, i.e.

$$
\begin{aligned}
\boldsymbol{u}_{\mathrm{c}} & =\boldsymbol{u}_{\mathrm{c}}^{M}+\boldsymbol{u}_{\mathrm{c}}^{s}, \\
u_{\mathrm{s}, 1} & =u_{\mathrm{s}, 1}^{M}+u_{\mathrm{s}, 1}^{s}, \\
u_{\mathrm{s}, \perp} & =u_{\mathrm{s}, \perp}^{M}+u_{\mathrm{s}, \perp}^{s} .
\end{aligned}
$$

The proposed approach results in a large-scale problem in terms of a globally "smooth" field $\overline{\boldsymbol{u}} \neq \boldsymbol{u}_{\mathrm{c}}$ and a subscale problem inside each representative volume element (RVE). The RVE is defined in a subscale region $\Omega_{\square}$ with the boundary $\partial \Omega_{\square}=\Gamma_{\square}$, and its centre has the macroscopic location $\overline{\boldsymbol{x}}$.

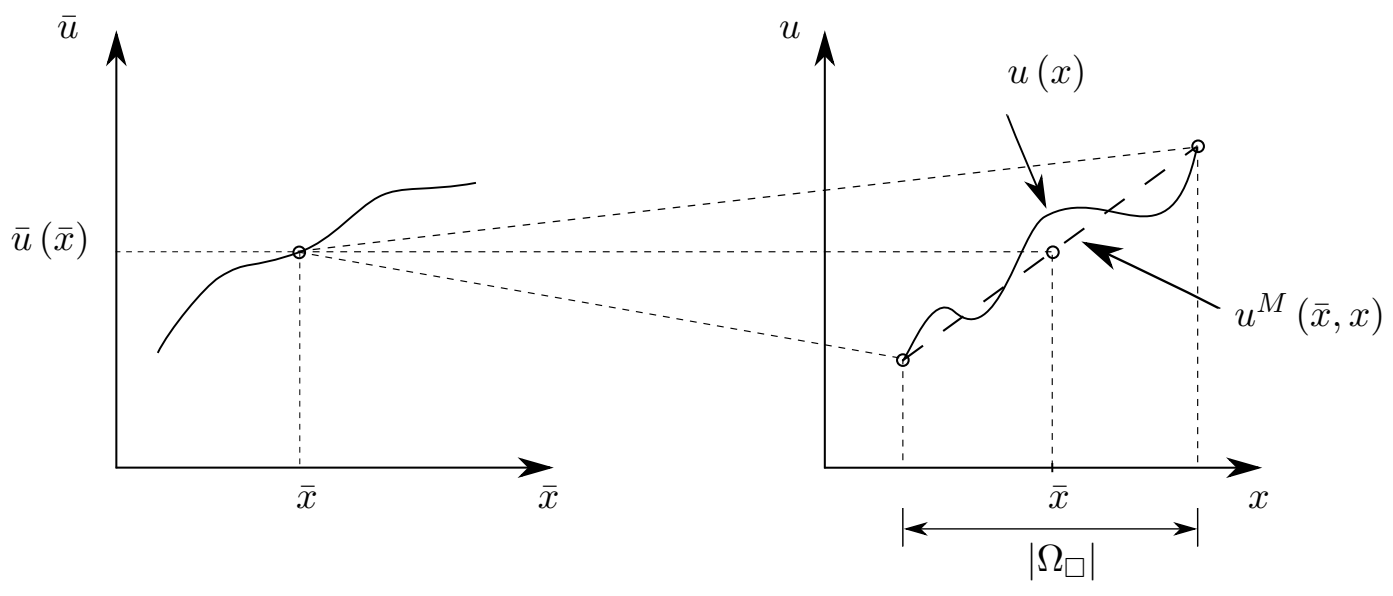

Figure 4: Separation of scales and illustration of assumed linear variation of $\boldsymbol{u}^{M}(\overline{\boldsymbol{x}} ; \boldsymbol{x})$.

The assumed scale separation is schematically depicted in Figure 4. Classical (first-order) computational homogenisation is adopted in this paper. For simplicity, it is assumed that the macroscopic parts of all the local fields derive from the same large-scale field $\overline{\boldsymbol{u}}$. Hence, they are expressed as:

$$
\begin{aligned}
\boldsymbol{u}_{\mathrm{c}}^{M} & =\overline{\boldsymbol{u}}(\overline{\boldsymbol{x}})+\left.[\overline{\boldsymbol{u}} \otimes \nabla]\right|_{\overline{\boldsymbol{x}}} \cdot[\boldsymbol{x}-\overline{\boldsymbol{x}}], \\
u_{\mathrm{s}, \mathrm{l}}^{M} & =\boldsymbol{e}_{l} \cdot \boldsymbol{u}_{\mathrm{c}}^{M}=\boldsymbol{e}_{l} \cdot \overline{\boldsymbol{u}}(\overline{\boldsymbol{x}})+\left.\boldsymbol{e}_{l} \cdot[\overline{\boldsymbol{u}} \otimes \nabla]\right|_{\overline{\boldsymbol{x}}} \cdot[\boldsymbol{x}-\overline{\boldsymbol{x}}], \\
u_{\mathrm{s}, \perp}^{M} & =\boldsymbol{e}_{\perp} \cdot \boldsymbol{u}_{\mathrm{c}}^{M}=\boldsymbol{e}_{\perp} \cdot \overline{\boldsymbol{u}}(\overline{\boldsymbol{x}})+\left.\boldsymbol{e}_{\perp} \cdot[\overline{\boldsymbol{u}} \otimes \nabla]\right|_{\overline{\boldsymbol{x}}} \cdot[\boldsymbol{x}-\overline{\boldsymbol{x}}]
\end{aligned}
$$

In the two-scale model setting, the local field is replaced by the homogenised field, i.e. at each location $\overline{\boldsymbol{x}} \in \Omega$ the field is approximated by the volume average on $\Omega_{\square}(\overline{\boldsymbol{x}})$. More specifically, for given functions $f_{\Omega}$ and $f_{\Gamma}$ defined on $\Omega_{\mathrm{c}}$ and $\Gamma_{\text {int }}$, respectively (see Figure 5 ), we have

$$
\int_{\Omega_{\mathrm{c}}} f_{\Omega} \mathrm{d} \Omega+\int_{\Gamma_{\mathrm{int}}} f_{\Gamma} \mathrm{d} \Gamma \mapsto \int_{\Omega} f_{\square} \mathrm{d} \Omega
$$

where the subscale average $f_{\square}$ is defined as:

$$
f_{\square}=\frac{1}{\left|\Omega_{\square}\right|}\left\{\int_{\Omega_{\square, \mathrm{c}}} f_{\Omega} \mathrm{d} \Omega+\int_{\Gamma_{\square, \mathrm{int}}} f_{\Gamma} \mathrm{d} \Gamma\right\} .
$$




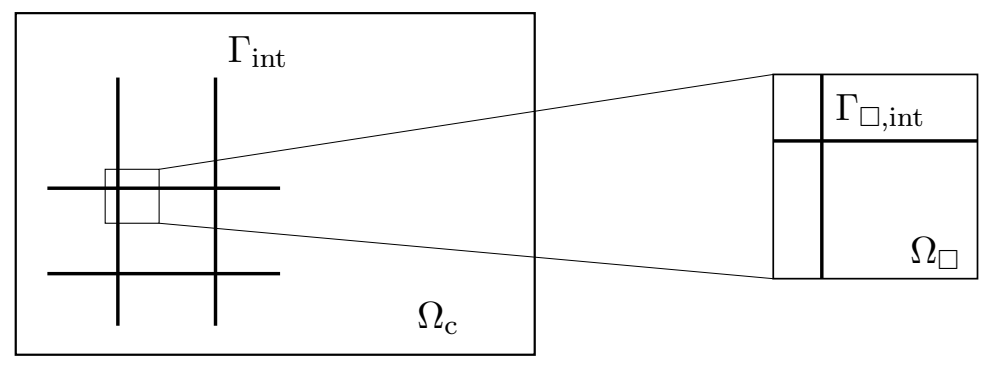

Figure 5: subscale region $\Omega_{\square}$ within the region $\Omega_{\mathrm{c}}$ along with the functions $f_{\Omega}$ and $f_{\Gamma}$ defined on regions $\Omega_{\mathrm{c}}$ and $\Gamma_{\text {int }}$ respectively.

The forms (23)-(28) can be expressed as the integral of the subscale averages. For example, $a_{\mathrm{c}}\left(\boldsymbol{u}_{\mathrm{c}} ; \delta \boldsymbol{u}_{\mathrm{c}}\right)$ is replaced by

$$
a_{\mathrm{c}}\left(\boldsymbol{u}_{\mathrm{c}} ; \delta \boldsymbol{u}_{\mathrm{c}}\right) \mapsto \int_{\Omega} a_{\square, \mathrm{c}}\left(\boldsymbol{u}_{\mathrm{c}} ; \delta \boldsymbol{u}_{\mathrm{c}}\right) \mathrm{d} \Omega,
$$

The fully resolved problem defined in (19)-(22) can then be rewritten as

$$
\begin{aligned}
\int_{\Omega}\left[a_{\square, \mathrm{c}}\left(\boldsymbol{u}_{\mathrm{c}} ; \delta \boldsymbol{u}_{\mathrm{c}}\right)\right. & +a_{\square, 1}\left(u_{\mathrm{s}, 1}, u_{\mathrm{s}, \perp} ; \delta u_{\mathrm{s}, \mathrm{l}}\right)+a_{\square, \mathrm{b}}\left(u_{\mathrm{s}, 1}, u_{\mathrm{s}, \perp} ; \delta u_{\mathrm{s}, \perp}\right)+c_{\square}\left(\lambda ; \delta u_{\mathrm{s}, \perp}-\boldsymbol{e}_{\perp} \cdot \delta \boldsymbol{u}_{\mathrm{c}}\right) \\
& \left.+b_{\square}\left(u_{\mathrm{s}, 1}-\boldsymbol{e}_{\mathrm{l}} \cdot \boldsymbol{u}_{\mathrm{c}} ; \delta u_{\mathrm{s}, 1}-\boldsymbol{e}_{\mathrm{l}} \cdot \delta \boldsymbol{u}_{\mathrm{c}}\right)+c_{\square}\left(\delta \lambda ; u_{\mathrm{s}, \perp}-\boldsymbol{e}_{\perp} \cdot \boldsymbol{u}_{\mathrm{c}}\right)\right] \mathrm{d} \Omega=l_{\mathrm{c}}\left(\delta \boldsymbol{u}_{\mathrm{c}}\right),
\end{aligned}
$$

where we introduced the RVE-forms

$$
\begin{aligned}
a_{\square, \mathrm{c}}\left(\boldsymbol{u}_{\mathrm{c}} ; \delta \boldsymbol{u}_{\mathrm{c}}\right) & :=\frac{1}{\left|\Omega_{\square}\right|} \int_{\Omega_{\square, \mathrm{c}}} t_{\mathrm{c}} \boldsymbol{\sigma}_{\mathrm{c}}\left(\boldsymbol{\varepsilon}\left[\boldsymbol{u}_{\mathrm{c}}\right]\right):\left[\delta \boldsymbol{u}_{\mathrm{c}} \otimes \nabla\right] \mathrm{d} \Omega, \\
b_{\square}(v ; w) & :=\frac{1}{\left|\Omega_{\square}\right|} \int_{\Gamma_{\square, \mathrm{int}}} S_{\mathrm{s}} t_{\Gamma}(v) w \mathrm{~d} \Gamma, \\
c_{\square}(\lambda ; w) & :=\frac{1}{\left|\Omega_{\square}\right|} \int_{\Gamma_{\square, \mathrm{int}}} \lambda v \mathrm{~d} \Gamma, \\
a_{\square, \mathrm{l}}\left(u_{\mathrm{s}, 1}, u_{\mathrm{s}, \perp} ; \delta u_{\mathrm{s}, 1}\right) & :=\frac{1}{\left|\Omega_{\square}\right|} \int_{\Gamma_{\square, \mathrm{int}}} N_{\mathrm{s}}\left(\frac{\partial u_{\mathrm{s}, 1}}{\partial l}, \frac{\partial^{2} u_{\mathrm{s}, \perp}}{\partial l^{2}}\right) \frac{\partial \delta u_{\mathrm{s}, 1}}{\partial l} \mathrm{~d} \Gamma, \\
a_{\square, \mathrm{b}}\left(u_{\mathrm{s}, 1}, u_{\mathrm{s}, \perp} ; \delta u_{\mathrm{s}, \perp}\right) & :=\frac{1}{\left|\Omega_{\square}\right|} \int_{\Gamma_{\square, \mathrm{int}}}-M_{\mathrm{s}}\left(\frac{\partial u_{\mathrm{s}, 1}}{\partial l}, \frac{\partial^{2} u_{\mathrm{s}, \perp}}{\partial l^{2}}\right) \frac{\partial^{2} \delta u_{\mathrm{s}, \perp}}{\partial l^{2}} \mathrm{~d} \Gamma .
\end{aligned}
$$

\subsection{Large-scale problem}

The VMS ansatz, formulated with (29)-(31), is introduced in (38), and the fully resolved problem can be split into two: the large-scale problem associated with "macroscopic" test functions $\delta \boldsymbol{u}_{\mathrm{c}}^{M}, \delta u_{\mathrm{s}, 1}^{M}, \delta u_{\mathrm{s}, \perp}^{M}$, and the subscale problem associated with the test functions $\delta \boldsymbol{u}_{\mathrm{c}}^{s}, \delta u_{\mathrm{s}, 1}^{s}, \delta u_{\mathrm{s}, \perp}^{s}, \delta \lambda$. In this section we consider the "macroscopic" problem, i.e.

$$
\begin{aligned}
\int_{\Omega}\left[a_{\square, \mathrm{c}}\left(\bullet ; \delta \boldsymbol{u}_{\mathrm{c}}^{M}\right)+b_{\square}\left(\bullet ; \delta u_{\mathrm{s}, \mathrm{l}}^{M}-\boldsymbol{e}_{\mathrm{l}} \cdot \delta \boldsymbol{u}_{\mathrm{c}}^{M}\right)+c_{\square}\left(\bullet ; \delta u_{\mathrm{s}, \perp}^{M}-\boldsymbol{e}_{\perp} \cdot \delta \boldsymbol{u}_{\mathrm{c}}^{M}\right)+a_{\square, l}\left(\bullet ; \delta u_{\mathrm{s}, 1}^{M}\right)\right. \\
\left.+a_{\square, \mathrm{b}}\left(\bullet ; \delta u_{\mathrm{s}, \perp}^{M}\right)\right] \mathrm{d} \Omega=l_{\mathrm{c}}\left(\delta \boldsymbol{u}_{\mathrm{c}}^{M}\right) .
\end{aligned}
$$


Based on the prolongation conditions (32)-(34), we define variational operators that are used in the subsequent derivation:

$$
\begin{aligned}
\delta \boldsymbol{u}_{\mathrm{c}}^{M} & =\left.\delta \overline{\boldsymbol{u}}\right|_{\overline{\boldsymbol{x}}}+\left.[\delta \overline{\boldsymbol{u}} \otimes \boldsymbol{\nabla}]\right|_{\overline{\boldsymbol{x}}} \cdot[\boldsymbol{x}-\overline{\boldsymbol{x}}], \\
\delta \boldsymbol{u}_{\mathrm{c}}^{M} \otimes \boldsymbol{\nabla} & =\left.[\delta \overline{\boldsymbol{u}} \otimes \boldsymbol{\nabla}]\right|_{\overline{\boldsymbol{x}}}, \\
\delta u_{\mathrm{s}, 1}^{M} & =\boldsymbol{e}_{l} \cdot \delta \boldsymbol{u}_{\mathrm{c}}^{M}=\left.\boldsymbol{e}_{l} \cdot \delta \overline{\boldsymbol{u}}\right|_{\overline{\boldsymbol{x}}}+\left.\boldsymbol{e}_{\mathrm{l}} \cdot[\delta \overline{\boldsymbol{u}} \otimes \nabla]\right|_{\overline{\boldsymbol{x}}} \cdot[\boldsymbol{x}-\overline{\boldsymbol{x}}], \\
\frac{\partial \delta u_{\mathrm{s}, 1}^{M}}{\partial l} & =\left[\delta u_{\mathrm{s}, 1}^{M} \otimes \nabla\right] \cdot \boldsymbol{e}_{1}=\left.[\delta \overline{\boldsymbol{u}} \otimes \boldsymbol{\nabla}]\right|_{\overline{\boldsymbol{x}}}:\left[\boldsymbol{e}_{\mathrm{l}} \otimes \boldsymbol{e}_{\mathrm{l}}\right], \\
\delta u_{\mathrm{s}, \perp}^{M} & =\boldsymbol{e}_{\perp} \cdot \delta \boldsymbol{u}_{\mathrm{c}}^{M}=\left.\boldsymbol{e}_{\perp} \cdot \delta \overline{\boldsymbol{u}}\right|_{\overline{\boldsymbol{x}}}+\left.\boldsymbol{e}_{\perp} \cdot[\delta \overline{\boldsymbol{u}} \otimes \boldsymbol{\nabla}]\right|_{\overline{\boldsymbol{x}}} \cdot[\boldsymbol{x}-\overline{\boldsymbol{x}}], \\
\frac{\partial \delta u_{\mathrm{s}, \perp}^{M}}{\partial l} & =\left[\delta u_{\mathrm{s}, \perp}^{M} \otimes \boldsymbol{\nabla}\right] \cdot \boldsymbol{e}_{\mathrm{l}}=\left.[\delta \overline{\boldsymbol{u}} \otimes \boldsymbol{\nabla}]\right|_{\overline{\boldsymbol{x}}}:\left[\boldsymbol{e}_{\perp} \otimes \boldsymbol{e}_{\mathrm{l}}\right], \\
\frac{\partial^{2} \delta u_{\mathrm{s}, \perp}^{M}}{\partial l^{2}} & =0 .
\end{aligned}
$$

Moreover, it is assumed that the local field is sufficiently smooth along the external boundary $\Gamma_{\text {ext }}$, and can successfully be approximated by the macroscopic field, i.e.

$$
l_{\mathrm{c}}\left(\delta \boldsymbol{u}_{\mathrm{c}}^{M}\right) \approx l_{\mathrm{c}}(\delta \overline{\boldsymbol{u}}) .
$$

With (45)-(51) and the approximation (52), the large-scale problem is obtained from (44) as

Find $\overline{\boldsymbol{u}} \in \overline{\mathbb{U}}$ such that

$$
\int_{\Omega} \overline{\boldsymbol{\sigma}}:[\delta \overline{\boldsymbol{u}} \otimes \nabla] \mathrm{d} \Omega=\int_{\Gamma_{\mathrm{ext}}} t_{\mathrm{c}} \hat{\boldsymbol{t}} \cdot \delta \overline{\boldsymbol{u}} \mathrm{d} \Gamma \quad \forall \delta \overline{\boldsymbol{u}} \in \overline{\mathbb{U}}^{0}
$$

with the suitable trial and test spaces

$$
\begin{aligned}
& \overline{\mathbb{U}}=\left\{\boldsymbol{u}(\boldsymbol{x}): \Omega \mapsto \mathbb{R}^{2}, \int_{\Omega} \boldsymbol{u}^{2}+[\boldsymbol{u} \otimes \nabla]^{2} \mathrm{~d} \Omega<\infty, \boldsymbol{u}=\boldsymbol{u}_{\mathrm{p}} \text { on } \Gamma_{\mathrm{p}}\right\}, \\
& \overline{\mathbb{U}}^{0}=\left\{\boldsymbol{u}(\boldsymbol{x}): \Omega \mapsto \mathbb{R}^{2}, \int_{\Omega} \boldsymbol{u}^{2}+[\boldsymbol{u} \otimes \nabla]^{2} \mathrm{~d} \Omega<\infty, \boldsymbol{u}=\mathbf{0} \text { on } \Gamma_{\mathrm{p}}\right\} .
\end{aligned}
$$

In (53), the macroscopic stress $\overline{\boldsymbol{\sigma}}$ is defined as

$$
\overline{\boldsymbol{\sigma}}=\frac{1}{\left|\Omega_{\square}\right|}\left\{\int_{\Omega_{\square, \mathrm{c}}} t_{\mathrm{c}} \boldsymbol{\sigma}_{\mathrm{c}} \mathrm{d} \Omega+\int_{\Gamma_{\square, \mathrm{int}}} N_{\mathrm{s}} \boldsymbol{e}_{l} \otimes \boldsymbol{e}_{\mathrm{l}} \mathrm{d} \Gamma\right\} .
$$

In the two-dimensional setting, the macroscopic stress $\overline{\boldsymbol{\sigma}}$ represents the large-scale membrane forces, i.e. its unit is force/length.

\section{Remark:}

By the divergence theorem, the macroscopic stress $\overline{\boldsymbol{\sigma}}$ can be computed from the subscale problem for the RVE as a result of computational homogenisation in the region $\Omega_{\square}$ as well as along the 
boundary $\Gamma_{\square}$ :

$$
\begin{array}{r}
\overline{\boldsymbol{\sigma}}=\frac{1}{\left|\Omega_{\square}\right|}\left\{\int_{\Gamma_{\square}} t_{\mathrm{c}} \hat{\boldsymbol{t}} \otimes[\boldsymbol{x}-\overline{\boldsymbol{x}}] \mathrm{d} \Gamma+\sum_{\Gamma_{\square, \mathrm{int}} \cap \Gamma_{\square}}\left[\hat{R}_{\mathrm{L}} \boldsymbol{e}_{1}+\hat{R}_{\perp} \boldsymbol{e}_{\perp}\right] \otimes[\boldsymbol{x}-\overline{\boldsymbol{x}}]\right. \\
\left.+\sum_{\Gamma_{\square, \mathrm{int}} \cap \Gamma_{\square}} \hat{R}_{\mathrm{M}} \boldsymbol{e}_{\perp} \otimes \boldsymbol{e}_{\mathrm{l}}\right\}
\end{array}
$$

where the summation on the discrete forces $\hat{R}_{\mathrm{L}}, \hat{R}_{\perp}$ and $\hat{R}_{\mathrm{M}}$ pertains to the points where the reinforcement intersects $\Gamma_{\square}$, cf. Figure 6.

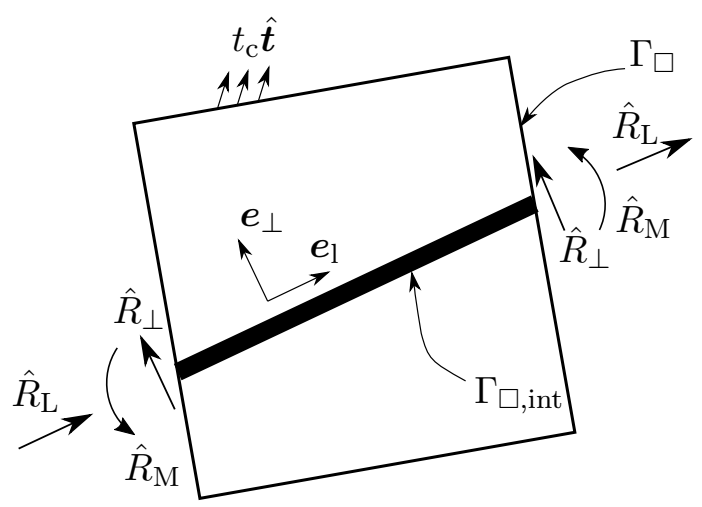

Figure 6: Discrete forces and traction at the boundary $\Gamma_{\square}$ of a RVE.

These forces together with the boundary traction $\hat{\boldsymbol{t}}$ are defined as follows:

$$
\begin{aligned}
& t_{\mathrm{c}} \boldsymbol{\sigma}_{\mathrm{c}} \cdot \boldsymbol{n}=t_{\mathrm{c}} \hat{\boldsymbol{t}} \quad \text { on } \Gamma_{\square}, \\
& N_{\mathrm{s}} e_{\ln }=\hat{R}_{\mathrm{L}} \quad \text { on } \Gamma_{\square} \cap \Gamma_{\square, \text { int }}, \\
& T_{\mathrm{s}} e_{\ln }=\hat{R}_{\perp} \quad \text { on } \Gamma_{\square} \cap \Gamma_{\square, \text { int }} \text {, } \\
& -M_{\mathrm{s}} e_{\mathrm{ln}}=\hat{R}_{\mathrm{M}} \quad \text { on } \Gamma_{\square} \cap \Gamma_{\square, \text { int }},
\end{aligned}
$$

with the scalar $e_{\ln }$ defined as

$$
e_{\mathrm{ln}}= \begin{cases}1 & \text { if } \boldsymbol{n} \cdot \boldsymbol{e}_{1}>0 \\ -1 & \text { if } \boldsymbol{n} \cdot \boldsymbol{e}_{1}<0\end{cases}
$$

\subsection{Subscale problem}

The RVE problem is obtained from (38) by restricting the test functions to the fluctuation fields $\delta \boldsymbol{u}_{\mathrm{c}}^{s}, \delta u_{\mathrm{s}, 1}^{s}, \delta u_{\mathrm{s}, \perp}^{s}$ and $\delta \lambda$. In general, before specifying the boundary conditions on any given RVE, subscale equilibrium can be stated as follows:

$$
\begin{array}{rlrl}
a_{\square, \mathrm{c}}\left(\boldsymbol{u}_{\mathrm{c}} ; \delta \boldsymbol{u}_{\mathrm{c}}^{s}\right)-b_{\square}\left(u_{\mathrm{s}, 1}-\boldsymbol{e}_{\mathrm{l}} \cdot \boldsymbol{u}_{\mathrm{c}} ; \boldsymbol{e}_{\mathrm{l}} \cdot \delta \boldsymbol{u}_{\mathrm{c}}^{s}\right) & \\
-c_{\square}\left(\lambda ; \boldsymbol{e}_{\perp} \cdot \delta \boldsymbol{u}_{\mathrm{c}}^{s}\right) & =l_{\square, \mathrm{c}}\left(\delta \boldsymbol{u}_{\mathrm{c}}^{s}\right) & \forall \delta \boldsymbol{u}_{\mathrm{c}}^{s} \in \mathbb{U}_{\square, \mathrm{c}}, \\
a_{\square, 1}\left(u_{\mathrm{s}, \mathrm{l}}, u_{\mathrm{s}, \perp} ; \delta u_{\mathrm{s}, 1}^{s}\right)+b_{\square}\left(u_{\mathrm{s}, 1}-\boldsymbol{e}_{\mathrm{l}} \cdot \boldsymbol{u}_{\mathrm{c}} ; \delta u_{\mathrm{s}, 1}^{s}\right) & =l_{\square, \mathrm{l}}\left(\delta u_{\mathrm{s}, 1}^{s}\right) & \forall \delta u_{\mathrm{s}, 1}^{s} \in \mathbb{U}_{\square, \mathrm{s}, 1}, \\
a_{\square, \mathrm{b}}\left(u_{\mathrm{s}, 1}, u_{\mathrm{s}, \perp} ; \delta u_{\mathrm{s}, \perp}^{s}\right)+c_{\square}\left(\lambda ; \delta u_{\mathrm{s}, \perp}^{s}\right) & =l_{\square, \mathrm{b}}\left(\delta u_{\mathrm{s}, \perp}^{s}\right) & \forall u_{\mathrm{s}, \perp}^{s} \in \mathbb{U}_{\square, \mathrm{s}, \perp}, \\
c_{\square}\left(\delta \lambda ; u_{\mathrm{s}, \perp}-\boldsymbol{e}_{\perp} \cdot \boldsymbol{u}_{\mathrm{c}}\right) & =0 & \forall \delta \lambda \in \mathbb{L}_{\square},
\end{array}
$$


with the test function spaces defined as:

$$
\begin{aligned}
& \mathbb{U}_{\square, \mathrm{c}}=\left\{\boldsymbol{u}(\boldsymbol{x}): \Omega_{\square} \mapsto \mathbb{R}^{2}, \int_{\Omega_{\square}} \boldsymbol{u}^{2}+[\boldsymbol{u} \otimes \boldsymbol{\nabla}]^{2} \mathrm{~d} \Omega<\infty\right\}, \\
& \mathbb{U}_{\square, \mathrm{s}, \mathrm{l}}=\left\{v(l): \Gamma_{\square, \text { int }} \mapsto \mathbb{R}, \int_{\Gamma_{\square, \mathrm{int}}} v^{2}+\left(\frac{\partial v}{\partial l}\right)^{2} \mathrm{~d} \Gamma<\infty\right\}, \\
& \mathbb{U}_{\square, \mathrm{s}, \perp}=\left\{v(l): \Gamma_{\square, \text { int }} \mapsto \mathbb{R}, \int_{\Gamma_{\square \text { int }}} v^{2}+\left(\frac{\partial v}{\partial l}\right)^{2}+\left(\frac{\partial^{2} v}{\partial l^{2}}\right)^{2} \mathrm{~d} \Gamma<\infty\right\}, \\
& \mathbb{L}_{\square}=\left\{\lambda(l): \Gamma_{\square, \text { int }} \mapsto \mathbb{R}, \int_{\Gamma_{\square \text { int }}} \lambda^{2} \mathrm{~d} \Gamma<\infty\right\} .
\end{aligned}
$$

From the equilibrium relations in (61)-(64) it can easily be verified that the output, i.e. the macroscopic stress stated in (54), is invariant under superimposed rigid body motion. Hence, the required input from the macroscale to the subscale problem can be expressed as

$$
\begin{aligned}
\boldsymbol{u}_{\mathrm{c}}^{M} & =\overline{\boldsymbol{\varepsilon}} \cdot[\boldsymbol{x}-\overline{\boldsymbol{x}}], \\
u_{\mathrm{s}, 1}^{M} & =\boldsymbol{e}_{l} \cdot \overline{\boldsymbol{\varepsilon}} \cdot[\boldsymbol{x}-\overline{\boldsymbol{x}}], \\
u_{\mathrm{s}, \perp}^{M} & =\boldsymbol{e}_{\perp} \cdot \overline{\boldsymbol{\varepsilon}} \cdot[\boldsymbol{x}-\overline{\boldsymbol{x}}] .
\end{aligned}
$$

as opposed to (32)-(34), where $\overline{\boldsymbol{\varepsilon}}:=\left.[\overline{\boldsymbol{u}} \otimes \nabla]\right|_{\overline{\boldsymbol{x}}} ^{\text {sym }}$ is the macroscopic strain. Furthermore, in the general case (before boundary conditions have been specified), the boundary terms are given as

$$
\begin{aligned}
l_{\square, \mathrm{c}}\left(\delta \boldsymbol{u}_{\mathrm{c}}\right) & =\frac{1}{\left|\Omega_{\square}\right|} \int_{\Gamma_{\square}} t_{\mathrm{c}} \hat{\boldsymbol{t}} \cdot \delta \boldsymbol{u}_{\mathrm{c}} \mathrm{d} \Gamma, \\
l_{\square, \mathrm{l}}\left(\delta u_{\mathrm{s}, 1}\right) & =\frac{1}{\left|\Omega_{\square}\right|} \sum_{\Gamma_{\square, \mathrm{int}} \cap \Gamma_{\square}} \hat{R}_{L} \delta u_{\mathrm{s}, \mathrm{l}}, \\
l_{\square, \mathrm{b}}\left(\delta u_{\mathrm{s}, \perp}\right) & =\frac{1}{\left|\Omega_{\square}\right|} \sum_{\Gamma_{\square, \mathrm{int}} \cap \Gamma_{\square}} \hat{R}_{\perp} \delta u_{\mathrm{s}, \perp}+\hat{R}_{M} \frac{\partial \delta u_{\mathrm{s}, \perp}}{\partial l},
\end{aligned}
$$

where the discrete forces $\hat{R}_{L}, \hat{R}_{\perp}, \hat{R}_{M}$, and the traction $\hat{\boldsymbol{t}}$ were defined in Figure 6 . Although the above formulation maintains generality, it must be noted that boundary conditions on the local fields need to be specified in order to produce a solvable system. This issue is discussed next. It is possible to consider either a Dirichlet or a Neumann type of boundary condition for each material (concrete and steel), i.e. to prescribe the deformation or the traction, respectively. In this paper, four combinations are considered. (i) Firstly, the deformations (along with rotations for the rebars) at the boundary are prescribed for both concrete and steel, thus imposing a "Dirichlet-Dirichlet" (henceforth denoted DD) combination of boundary conditions. (ii) Secondly, the bars are allowed to slip at the ends, while the deformation at the boundary of concrete is prescribed. Hence, this imposes a "Dirichlet-Neumann" (henceforth denoted DN) combination of boundary conditions on the RVE. (iii) Thirdly, the tractions are prescribed at the boundary of the concrete, while the deformations (and rotations) are prescribed for the steel bars. Apparently, this choice constitutes a "NeumannDirichlet" (denoted ND) combination. (iv) Finally, the tractions are prescribed at the boundary of the concrete, while the steel bars are allowed to slip at the ends, thus creating a "NeumannNeumann" (denoted NN) combination of boundary conditions. All of the cases are schematically depicted in Figure 7. 
Dirichlet-Dirichlet

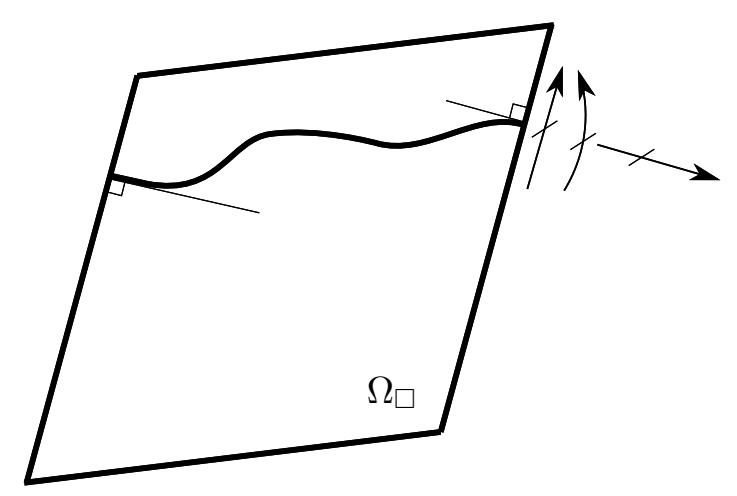

Neumann-Dirichlet

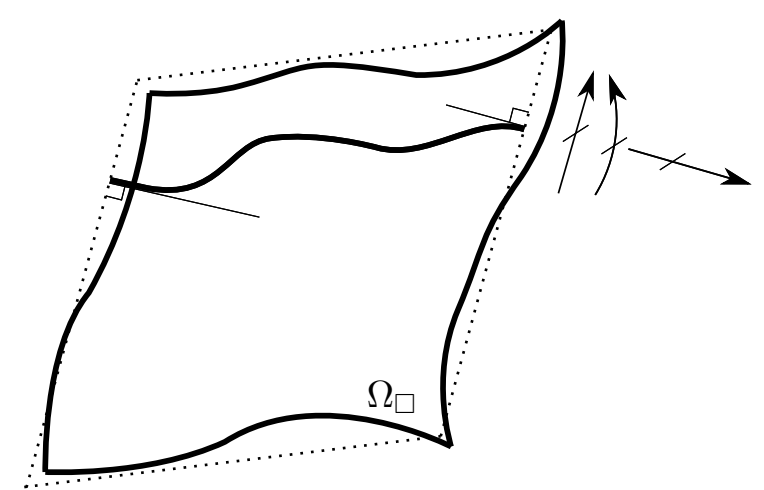

Dirichlet-Neumann

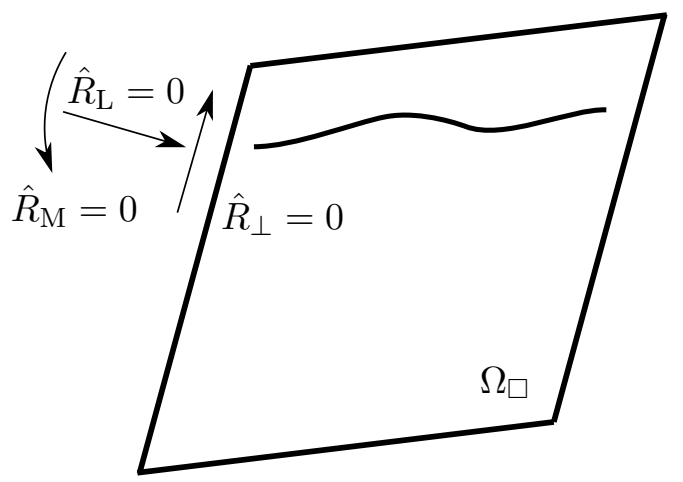

Neumann-Neumann

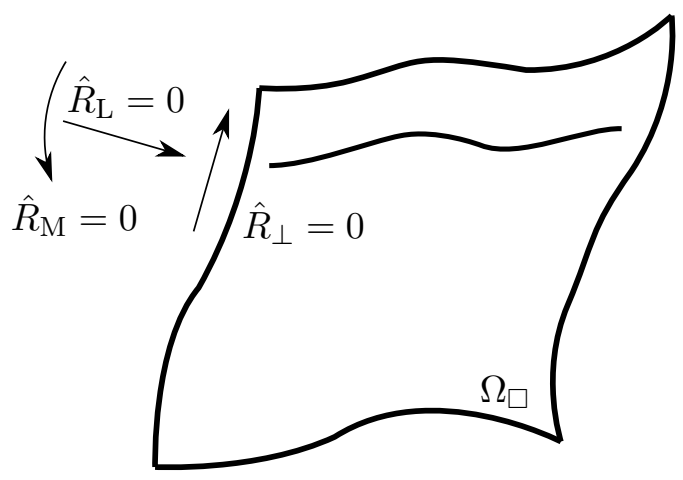

Figure 7: Dirichlet-Dirichlet, Dirichlet-Neumann, Neumann-Dirichlet and Neumann-Neumann combinations of boundary conditions for both fields in the RVE. The rebar is orthogonal to the boundary in the undeformed configuration.

\subsubsection{Dirichlet-Dirichlet boundary conditions}

The most straightforward choice is the homogeneous Dirichlet boundary condition on the fluctuation fields $\boldsymbol{u}_{\mathrm{c}}^{s}, u_{\mathrm{s}, 1}^{s}, u_{\mathrm{s}, \perp}^{s}$, whereby these fields vanish at the boundary $\Gamma_{\square}$. Thus, the local displacement fields of the reinforcing bars still derive from the macroscopic field in the concrete. The conditions can be expressed as:

$$
\begin{aligned}
\boldsymbol{u}_{\mathrm{c}} & =\boldsymbol{u}_{\mathrm{c}}^{M}=\overline{\boldsymbol{\varepsilon}} \cdot[\boldsymbol{x}-\overline{\boldsymbol{x}}] & & \text { on } \Gamma_{\square}, \\
u_{\mathrm{s}, 1} & =u_{\mathrm{s}, 1}^{M}=\boldsymbol{e}_{1} \cdot \overline{\boldsymbol{\varepsilon}} \cdot[\boldsymbol{x}-\overline{\boldsymbol{x}}] & & \text { on } \Gamma_{\square} \cap \Gamma_{\square, \mathrm{int}}, \\
u_{\mathrm{s}, \perp} & =u_{\mathrm{s}, \perp}^{M}=\boldsymbol{e}_{\perp} \cdot \overline{\boldsymbol{\varepsilon}} \cdot[\boldsymbol{x}-\overline{\boldsymbol{x}}] & & \text { on } \Gamma_{\square} \cap \Gamma_{\square, \text { int }}
\end{aligned}
$$

From the VMS ansatz it follows that:

$$
\begin{aligned}
\delta \boldsymbol{u}_{\mathrm{c}}^{s}=0 & & \text { on } \Gamma_{\square}, \\
\delta u_{\mathrm{s}, \mathrm{l}}^{s}=0 & & \text { on } \Gamma_{\square} \cap \Gamma_{\square, \text { int }}, \\
\delta u_{\mathrm{s}, \perp}^{s}=0 & & \text { on } \Gamma_{\square} \cap \Gamma_{\square, \text { int }} .
\end{aligned}
$$


Rather than solving for the local fluctuation fields $\boldsymbol{u}_{\mathrm{c}}^{s}(\boldsymbol{x}), u_{\mathrm{s}, 1}^{s}(l), u_{\mathrm{s}, \perp}^{s}(l)$, we may find it more feasible, from the computational point of view, to solve for the total local fields $\boldsymbol{u}_{\mathrm{c}}(\boldsymbol{x}), u_{\mathrm{s}, 1}(l)$, $u_{\mathrm{s}, \perp}(l)$. Upon expanding the subscale equilibrium conditions (61)-(64) and employing the Dirichlet boundary conditions, we note that the boundary terms on the right-hand side disappear, and the Dirichlet-Dirichlet subscale problem can be expressed as follows:

Find $\boldsymbol{u}_{\mathrm{c}}, u_{\mathrm{s}, \mathrm{l}}, u_{\mathrm{s}, \perp}, \lambda \in \mathbb{U}_{\square, \mathrm{c}}^{D}(\overline{\boldsymbol{\varepsilon}}) \times \mathbb{U}_{\square, \mathrm{s}, \mathrm{l}}^{D}(\overline{\boldsymbol{\varepsilon}}) \times \mathbb{U}_{\square, \mathrm{s}, \perp}^{D}(\overline{\boldsymbol{\varepsilon}}) \times \mathbb{L}_{\square}$ such that

$$
\begin{aligned}
& \int_{\Omega_{\square, \mathrm{c}}} t_{\mathrm{c}} \boldsymbol{\sigma}_{\mathrm{c}}:\left[\delta \boldsymbol{u}_{\mathrm{c}} \otimes \nabla\right] \mathrm{d} \Omega-\int_{\Gamma_{\square, \mathrm{int}}} S_{\mathrm{s}} t_{\Gamma} \boldsymbol{e}_{1} \cdot \delta \boldsymbol{u}_{\mathrm{c}} \mathrm{d} \Gamma \\
& -\int_{\Gamma_{\square, \mathrm{int}}} \lambda \boldsymbol{e}_{\perp} \cdot \delta \boldsymbol{u}_{\mathrm{c}} \mathrm{d} \Gamma=0 \quad \forall \delta \boldsymbol{u}_{\mathrm{c}} \in \mathbb{U}_{\square, \mathrm{c}}^{D}(\mathbf{0}), \\
& \int_{\Gamma_{\square, \mathrm{int}}} N_{\mathrm{s}} \frac{\partial \delta u_{\mathrm{s}, \mathrm{l}}}{\partial l} \mathrm{~d} \Gamma+\int_{\Gamma_{\square, \mathrm{int}}} S_{\mathrm{s}} t_{\Gamma} \delta u_{\mathrm{s}, \mathrm{l}} \mathrm{d} \Gamma=0 \quad \forall \delta u_{\mathrm{s}, l} \in \mathbb{U}_{\square, \mathrm{s}, \mathrm{l}}^{D}(\mathbf{0}), \\
& -\int_{\Gamma_{\square, \text { int }}} M_{\mathrm{s}} \frac{\partial^{2} \delta u_{\mathrm{s}, \perp}}{\partial l^{2}} \mathrm{~d} \Gamma+\int_{\Gamma_{\square, \mathrm{int}}} \lambda \delta u_{\mathrm{s}, \perp} \mathrm{d} \Gamma=0 \quad \forall \delta u_{\mathrm{s}, \perp} \in \mathbb{U}_{\square, \mathrm{s}, \perp}^{D}(\mathbf{0}), \\
& \int_{\Gamma_{\square, \text { int }}}\left[u_{\mathrm{s}, \perp}-\boldsymbol{e}_{\perp} \cdot \boldsymbol{u}_{\mathrm{c}}\right] \delta \lambda \mathrm{d} \Gamma=0 \quad \forall \delta \lambda \in \mathbb{L}_{\square},
\end{aligned}
$$

for suitable trial sets

$$
\begin{aligned}
\mathbb{U}_{\square, \mathrm{c}}^{D}(\overline{\boldsymbol{\varepsilon}}) & =\left\{\boldsymbol{u} \in \mathbb{U}_{\square, \mathrm{c}}: \boldsymbol{u}=\overline{\boldsymbol{\varepsilon}} \cdot[\boldsymbol{x}-\overline{\boldsymbol{x}}] \text { on } \Gamma_{\square}\right\}, \\
\mathbb{U}_{\square, \mathrm{s}, \mathrm{l}}^{D}(\overline{\boldsymbol{\varepsilon}}) & =\left\{v \in \mathbb{U}_{\square, \mathrm{s}, \mathrm{l}}: v=\boldsymbol{e}_{\mathrm{l}} \cdot \overline{\boldsymbol{\varepsilon}} \cdot[\boldsymbol{x}-\overline{\boldsymbol{x}}] \text { on } \Gamma_{\square} \cap \Gamma_{\square, \mathrm{int}}\right\}, \\
\mathbb{U}_{\square, \mathrm{s}, \perp}^{D}(\overline{\boldsymbol{\varepsilon}}) & =\left\{v \in \mathbb{U}_{\square, \mathrm{s}, \perp}: v=\boldsymbol{e}_{\perp} \cdot \overline{\boldsymbol{\varepsilon}} \cdot[\boldsymbol{x}-\overline{\boldsymbol{x}}], \text { and } \frac{\partial v}{\partial l}=\overline{\boldsymbol{\varepsilon}}:\left[\boldsymbol{e}_{\perp} \otimes \boldsymbol{e}_{\mathrm{l}}\right] \text { on } \Gamma_{\square} \cap \Gamma_{\square, \text { int }}\right\},
\end{aligned}
$$

and the pertinent test spaces.

\subsubsection{Dirichlet-Neumann boundary conditions}

Prescribing translations and rotations of the reinforcing bars at the boundary $\Gamma_{\square}$ will give rise to reactions (forces and moments) at the end points $\Gamma_{\text {int }} \cap \Gamma_{\square \text {,int }}$, see Figure 7 . These variationally consistent reactions can be retrieved in a postprocessing step. An alternative possibility is to require that the reaction forces $\hat{R}_{\mathrm{L}}, \hat{R}_{\perp}, \hat{R}_{\mathrm{M}}$ vanish. The Neumann boundary conditions for the reinforcement are thus expressed as:

$$
\hat{R}_{\mathrm{L}}=\hat{R}_{\perp}=0, \quad \hat{R}_{\mathrm{M}}=0 \quad \text { on } \Gamma_{\square} \cap \Gamma_{\square, \text { int }} .
$$

Contrary to the DD case described above, the local fluctuations $u_{\mathrm{s}, 1}^{s}(\boldsymbol{x})$ and $u_{\mathrm{s}, \perp}^{s}(\boldsymbol{x})$ on the boundary are no longer constrained, i.e. the bars are now free to move at the ends (relative to concrete). For the sake of completeness, the corresponding subscale problem with Dirichlet-Neumann boundary conditions is given as: 
Find $\boldsymbol{u}_{\mathrm{c}}, u_{\mathrm{s}, 1}, u_{\mathrm{s}, \perp}, \lambda \in \mathbb{U}_{\square, \mathrm{c}}^{D}(\overline{\boldsymbol{\varepsilon}}) \times \mathbb{U}_{\square, \mathrm{s}, 1} \times \mathbb{U}_{\square, \mathrm{s}, \perp} \times \mathbb{L}_{\square}$ such that

$$
\begin{aligned}
& \int_{\Omega_{\square, \mathrm{c}}} t_{\mathrm{c}} \boldsymbol{\sigma}_{\mathrm{c}}:\left[\delta \boldsymbol{u}_{\mathrm{c}} \otimes \nabla\right] \mathrm{d} \Omega-\int_{\Gamma_{\square, \mathrm{int}}} S_{\mathrm{s}} t_{\Gamma} \boldsymbol{e}_{\mathrm{l}} \cdot \delta \boldsymbol{u}_{\mathrm{c}} \mathrm{d} \Gamma \\
& +\int_{\Gamma_{\square, \mathrm{int}}} \lambda \boldsymbol{e}_{\perp} \cdot \delta \boldsymbol{u}_{\mathrm{c}} \mathrm{d} \Gamma=0 \quad \forall \delta \boldsymbol{u}_{\mathrm{c}} \in \mathbb{U}_{\square, \mathrm{c}}^{D}(\mathbf{0}), \\
& \int_{\Gamma_{\square, \mathrm{int}}} N_{\mathrm{s}} \frac{\partial \delta u_{\mathrm{s}, \mathrm{l}}}{\partial l} \mathrm{~d} \Gamma+\int_{\Gamma_{\square, \mathrm{int}}} S_{\mathrm{s}} t_{\Gamma} \delta u_{\mathrm{s}, \mathrm{l}} \mathrm{d} \Gamma=0 \quad \forall \delta u_{\mathrm{s}, 1} \in \mathbb{U}_{\square, \mathrm{s}, \mathrm{l}}, \\
& -\int_{\Gamma_{\square, \mathrm{int}}} M_{\mathrm{s}} \frac{\partial^{2} \delta u_{\mathrm{s}, \perp}}{\partial l^{2}} \mathrm{~d} \Gamma+\int_{\Gamma_{\square, \mathrm{int}}} \lambda \delta u_{\mathrm{s}, \perp} \mathrm{d} \Gamma=0 \quad \forall \delta u_{\mathrm{s}, \perp} \in \mathbb{U}_{\square, \mathrm{s}, \perp}, \\
& \int_{\Gamma_{\square, \mathrm{int}}}\left[u_{\mathrm{s}, \perp}-\boldsymbol{e}_{\perp} \cdot \boldsymbol{u}_{\mathrm{c}}\right] \delta \lambda \mathrm{d} \Gamma=0 \quad \forall \delta \lambda \in \mathbb{L}_{\square},
\end{aligned}
$$

for previously introduced trial sets and test spaces $\mathbb{U}_{\square, \mathrm{c}}^{D}(\overline{\boldsymbol{\varepsilon}}), \mathbb{U}_{\square, \mathrm{c}}^{D}(\mathbf{0}), \mathbb{U}_{\square, \mathrm{s}, \mathrm{l}}, \quad \mathbb{U}_{\square, \mathrm{s}, \perp}, \mathbb{L}_{\square}$.

\subsubsection{Neumann-Dirichlet boundary conditions}

It is assumed that the boundary tractions on the RVE are generated by a constant macroscopic stress tensor $\hat{\boldsymbol{\sigma}}_{\mathrm{c}}$. This can be easily done if the problem under considerations is run in load control. However, this is often not the case, and some constraints must usually be used to resolve the problem. In case of displacement control, when a macroscopic strain tensor is prescribed on the RVE, such problem must be solved iteratively. In such situation, the constraint will assure that the volume average of strain in the RVE is equal to the imposed macroscopic strain. In our case, Lagrange multipliers can be used to impose the constraint. Introducing the constraint that the volume average of the strain in the concrete phase is equal to the macroscopic strain $\bar{\varepsilon}$, i.e.

$$
\frac{1}{\left|\Omega_{\square}\right|} \int_{\Omega_{\square, \mathrm{c}}}\left[\boldsymbol{u}_{\mathrm{c}} \otimes \boldsymbol{\nabla}\right]^{\mathrm{sym}} \mathrm{d} \Omega=\overline{\boldsymbol{\varepsilon}},
$$

we identify Lagrange multipliers as the components of the average concrete stress tensor $\hat{\boldsymbol{\sigma}}_{\mathrm{c}}$. Then, the subscale problem with Neumann-Dirichlet boundary conditions can be expressed as: 
For a given $\overline{\boldsymbol{\varepsilon}}$ find $\boldsymbol{u}_{\mathrm{c}}, u_{\mathrm{s}, \mathrm{l}}, u_{\mathrm{s}, \perp}, \lambda \in \mathbb{U}_{\square, \mathrm{c}}^{\mathrm{N}} \times \mathbb{U}_{\square, \mathrm{s}, \mathrm{l}}^{D}(\overline{\boldsymbol{\varepsilon}}) \times \mathbb{U}_{\square, \mathrm{s}, \perp}^{D}(\overline{\boldsymbol{\varepsilon}}) \times \mathbb{L}_{\square}$, and $\hat{\boldsymbol{\sigma}}_{\mathrm{c}} \in \mathbb{R}^{2 \times 2}$ such that

$$
\begin{array}{cl}
\int_{\Omega_{\square, \mathrm{c}}} t_{\mathrm{c}} \boldsymbol{\sigma}_{\mathrm{c}}:\left[\delta \boldsymbol{u}_{\mathrm{c}} \otimes \nabla\right] \mathrm{d} \Omega-\int_{\Gamma_{\square, \mathrm{int}}} S_{\mathrm{s}} t_{\Gamma} \boldsymbol{e}_{\mathrm{l}} \cdot \delta \boldsymbol{u}_{\mathrm{c}}+\lambda \boldsymbol{e}_{\perp} \cdot \delta \boldsymbol{u}_{\mathrm{c}} \mathrm{d} \Gamma \\
-\int_{\Omega_{\square, \mathrm{c}}}\left[\delta \boldsymbol{u}_{\mathrm{c}} \otimes \nabla\right] \mathrm{d} \Omega: \hat{\boldsymbol{\sigma}}_{\mathrm{c}}=0 & \forall \delta \boldsymbol{u}_{\mathrm{c}} \in \mathbb{U}_{\square, \mathrm{c}}^{\mathrm{N}}, \\
-\int_{\Omega_{\square, \mathrm{c}}}\left[\boldsymbol{u}_{\mathrm{c}} \otimes \nabla\right] \mathrm{d} \Omega: \delta \hat{\boldsymbol{\sigma}}_{\mathrm{c}}=-\overline{\boldsymbol{\varepsilon}}: \delta \hat{\boldsymbol{\sigma}}_{\mathrm{c}} \Omega_{\square} & \forall \delta \hat{\boldsymbol{\sigma}}_{\mathrm{c}} \in \mathbb{R}^{2 \times 2}, \\
\int_{\Gamma_{\square, \mathrm{int}}} N_{\mathrm{s}} \frac{\partial \delta u_{\mathrm{s}, 1}}{\partial l} \mathrm{~d} \Gamma+\int_{\Gamma_{\square, \mathrm{int}}} S_{\mathrm{s}} t_{\Gamma} \delta u_{\mathrm{s}, \mathrm{l}} \mathrm{d} \Gamma=0 & \forall \delta u_{\mathrm{s}, 1} \in \mathbb{U}_{\square, \mathrm{s}, l}^{D}(\mathbf{0}), \\
-\int_{\Gamma_{\square, \mathrm{int}}} M_{\mathrm{s}} \frac{\partial^{2} \delta u_{\mathrm{s}, \perp}}{\partial l^{2}} \mathrm{~d} \Gamma+\int_{\Gamma_{\square, \mathrm{int}}} \lambda \delta u_{\mathrm{s}, \perp} \mathrm{d} \Gamma=0 & \forall \delta u_{\mathrm{s}, \perp} \in \mathbb{U}_{\square, \mathrm{s}, \perp}^{D}(\mathbf{0}), \\
\int_{\Gamma_{\square, \mathrm{int}}}\left[u_{\mathrm{s}, \perp}-\boldsymbol{e}_{\perp} \cdot \boldsymbol{u}_{\mathrm{c}}\right] \delta \lambda \mathrm{d} \Gamma=0 & \forall \delta \lambda \in \mathbb{L}_{\square},
\end{array}
$$

where

$$
\mathbb{U}_{\square, \mathrm{c}}^{\mathrm{N}}=\left\{\boldsymbol{v} \in \mathbb{U}_{\square, \mathrm{c}}, \int_{\Omega_{\square}} v \mathrm{~d} \Omega=0\right\},
$$

while $\mathbb{U}_{\square, \mathrm{s}, \mathrm{l}}^{D}(\overline{\boldsymbol{\varepsilon}}), \mathbb{U}_{\square, \mathrm{s}, \perp}^{D}(\overline{\boldsymbol{\varepsilon}}), \mathbb{L}_{\square}, \mathbb{R}^{2 \times 2}, \mathbb{U}_{\square, \mathrm{s}, \mathrm{l}}^{D}(\mathbf{0}), \mathbb{U}_{\square, \mathrm{s}, \perp}^{D}(\mathbf{0})$ were defined previously.

\subsubsection{Neumann-Neumann boundary conditions}

Following the same reasoning as presented in 3.3.2 and 3.3.3, we can express the subscale problem with Neumann-Neumann boundary conditions as:

For a given $\overline{\boldsymbol{\varepsilon}}$ find $\boldsymbol{u}_{\mathrm{c}}, u_{\mathrm{s}, 1}, u_{\mathrm{s}, \perp}, \lambda \in \mathbb{U}_{\square, \mathrm{c}}^{\mathrm{N}} \times \mathbb{U}_{\square, \mathrm{s}, 1} \times \mathbb{U}_{\square, \mathrm{s}, \perp} \times \mathbb{L}_{\square}$, and $\hat{\boldsymbol{\sigma}}_{\mathrm{c}} \in \mathbb{R}^{2 \times 2}$ such that

$$
\begin{aligned}
& \int_{\Omega_{\square, \mathrm{c}}} t_{\mathrm{c}} \boldsymbol{\sigma}_{\mathrm{c}}:\left[\delta \boldsymbol{u}_{\mathrm{c}} \otimes \nabla\right] \mathrm{d} \Omega-\int_{\Gamma_{\square, \mathrm{int}}} S_{\mathrm{s}} t_{\Gamma} \boldsymbol{e}_{\mathrm{l}} \cdot \delta \boldsymbol{u}_{\mathrm{c}}+\lambda \boldsymbol{e}_{\perp} \cdot \delta \boldsymbol{u}_{\mathrm{c}} \mathrm{d} \Gamma \\
&-\int_{\Omega_{\square, \mathrm{c}}}\left[\delta \boldsymbol{u}_{\mathrm{c}} \otimes \boldsymbol{\nabla}\right] \mathrm{d} \Omega: \hat{\boldsymbol{\sigma}}_{\mathrm{c}}=0 \forall \delta \boldsymbol{u}_{\mathrm{c}} \in \mathbb{U}_{\square, \mathrm{c}}^{\mathrm{N}}, \\
&-\int_{\Omega_{\square, \mathrm{c}}}\left[\boldsymbol{u}_{\mathrm{c}} \otimes \nabla\right] \mathrm{d} \Omega: \delta \hat{\boldsymbol{\sigma}}_{\mathrm{c}}=-\overline{\boldsymbol{\varepsilon}}: \delta \hat{\boldsymbol{\sigma}}_{\mathrm{c}} \Omega_{\square} \forall \delta \hat{\boldsymbol{\sigma}}_{\mathrm{c}} \in \mathbb{R}^{2 \times 2}, \\
& \int_{\Gamma_{\square, \mathrm{int}}} N_{\mathrm{s}} \frac{\partial \delta u_{\mathrm{s}, 1}}{\partial l} \mathrm{~d} \Gamma+\int_{\Gamma_{\square, \mathrm{int}}} S_{\mathrm{s}} t_{\Gamma} \delta u_{\mathrm{s}, 1} \mathrm{~d} \Gamma=0 \forall \delta u_{\mathrm{s}, 1} \in \mathbb{U}_{\square, \mathrm{s}, l}, \\
&-\int_{\Gamma_{\square, \mathrm{int}}} M_{\mathrm{s}} \frac{\partial^{2} \delta u_{\mathrm{s}, \perp}}{\partial l^{2}} \mathrm{~d} \Gamma+\int_{\Gamma_{\square, \mathrm{int}}} \lambda \delta u_{\mathrm{s}, \perp} \mathrm{d} \Gamma=0 \forall \delta u_{\mathrm{s}, \perp} \in \mathbb{U}_{\square, \mathrm{s}, \perp}, \\
& \int_{\Gamma_{\square, \mathrm{int}}}\left[u_{\mathrm{s}, \perp}-\boldsymbol{e}_{\perp} \cdot \boldsymbol{u}_{\mathrm{c}}\right] \delta \lambda \mathrm{d} \Gamma=0 \forall \delta \lambda \in \mathbb{L}_{\square},
\end{aligned}
$$


for previously introduced trial and test spaces $\mathbb{U}_{\square, \mathrm{c}}^{\mathrm{N}}, \mathbb{U}_{\square, \mathrm{s}, \mathrm{l}}, \mathbb{U}_{\square, \mathrm{s}, \perp}, \mathbb{L}_{\square}, \mathbb{R}^{2 \times 2}$.

\section{Nested finite element algorithm}

Next, we consider the $\mathrm{FE}^{2}$-formulation, whereby the equations are discretized at both scales. To this end, we introduce the large-scale discretization $\overline{\mathbb{U}} \mapsto \overline{\mathbb{U}}_{\mathrm{h}}$ and the subscale discretizations $\mathbb{U}_{\square, \mathrm{c}} \mapsto \mathbb{U}_{\square, \mathrm{c}, \mathrm{h}}, \mathbb{U}_{\square, \mathrm{s}, \mathrm{l}} \mapsto \mathbb{U}_{\square, \mathrm{s}, \mathrm{l}, \mathrm{h}}, \mathbb{U}_{\square, \mathrm{s}, \perp} \mapsto \mathbb{U}_{\square, \mathrm{s}, \perp, \mathrm{h}}, \mathbb{L}_{\square} \mapsto \mathbb{L}_{\square, \mathrm{h}}$. The large-scale problem equation is evaluated at the quadrature points of the large-scale FE-mesh, with the effective stress tensor $\overline{\boldsymbol{\sigma}}_{\mathrm{h}}$ obtained from the subscale problem at these points. In each load step, after applying the displacement increment, the macroscopic strain tensor $\bar{\varepsilon}$ in each Gauss point is computed. This strain is imposed on the subscale unit cell either directly (via prescribed deformation) or indirectly (via Lagrange multipliers). The subscale problem, i.e. the discrete counterpart of Eqs. (77)-(80), (82)-(85), etc. is then solved, yielding the macroscopic stress tensor $\overline{\boldsymbol{\sigma}}$ (Eq. 55). After homogenisation of the stresses in each macroscopic finite element, the residual pertinent to (53) is computed for the whole large-scale model. The nested problem can be solved by Newton iterations on the large-scale. In order to do so, macroscopic tangent operator needs to be computed from the subscale problem. A few options are available: Firstly, it can be "extracted" from the tangent stiffness of the subscale RVE, cf. [6] or [22] for details. Secondly, if the subscale tangent stiffness cannot be accessed (e.g. due to using a commercial code), the consistent macroscopic tangent stiffness can be retrieved by numerical perturbation scheme, which can prove computationally expensive. Another alternative is to employ another iterative method on the large-scale, e.g. a Quasi-Newton BFGS method or even Modified Newton method can prove effective. For the first approximation, the linear elastic tangent stiffness can be extracted for the RVEs "offline". A schematic flowchart of the described procedure is illustrated in Figure 8.

\section{$5 \quad$ Effective subscale response}

\subsection{Preliminaries}

In order to investigate the effective response of reinforced concrete at the subscale, a few studies were considered. First, the elastic bounds on the stiffness were computed and studied for a number of RVEs (Section 5.3). Secondly, a series of tensile tests on the RVEs were simulated in order to study the effective response of reinforced concrete unit cells subjected to different combinations of boundary conditions (Section 5.4). For the simulations, the RVEs were modelled in the open source $\mathrm{C}++$ code OOFEM (www. oofem.org) [23].

\subsection{Modelling choices}

A periodic arrangement of reinforcement was considered for structuring any given RVE (or rather, a unit cells that is smaller than an RVE), which comprises the concrete continuum, reinforcement (longitudinal and transverse) and the steel/concrete interface. Each square unit cell contained one or more reinforcing bars in both directions. Rebars with diameter $\phi_{1}$ and $\phi_{2}$ were considered for horizontal and vertical reinforcement, respectively. The spacing of the bars $(200 \mathrm{~mm})$ was uniform in both directions. Three different RVEs were considered, see Figure 9. Depending on the number of the reinforcing bars in each direction, the unit cells are denoted " $1 \times 1$ ", " $2 \times 2$ " and " $3 \times 3$ ", respectively.

Regarding geometry, longitudinal $\phi_{1}=20 \mathrm{~mm}$ bars were chosen and $\phi_{2}=8 \mathrm{~mm}$ bars were considered for transverse reinforcement. The thickness of the RVEs was $0.2 \mathrm{~m}$. As for materials, a few choices were made. All the material parameters for concrete, reinforcing steel as well as the 


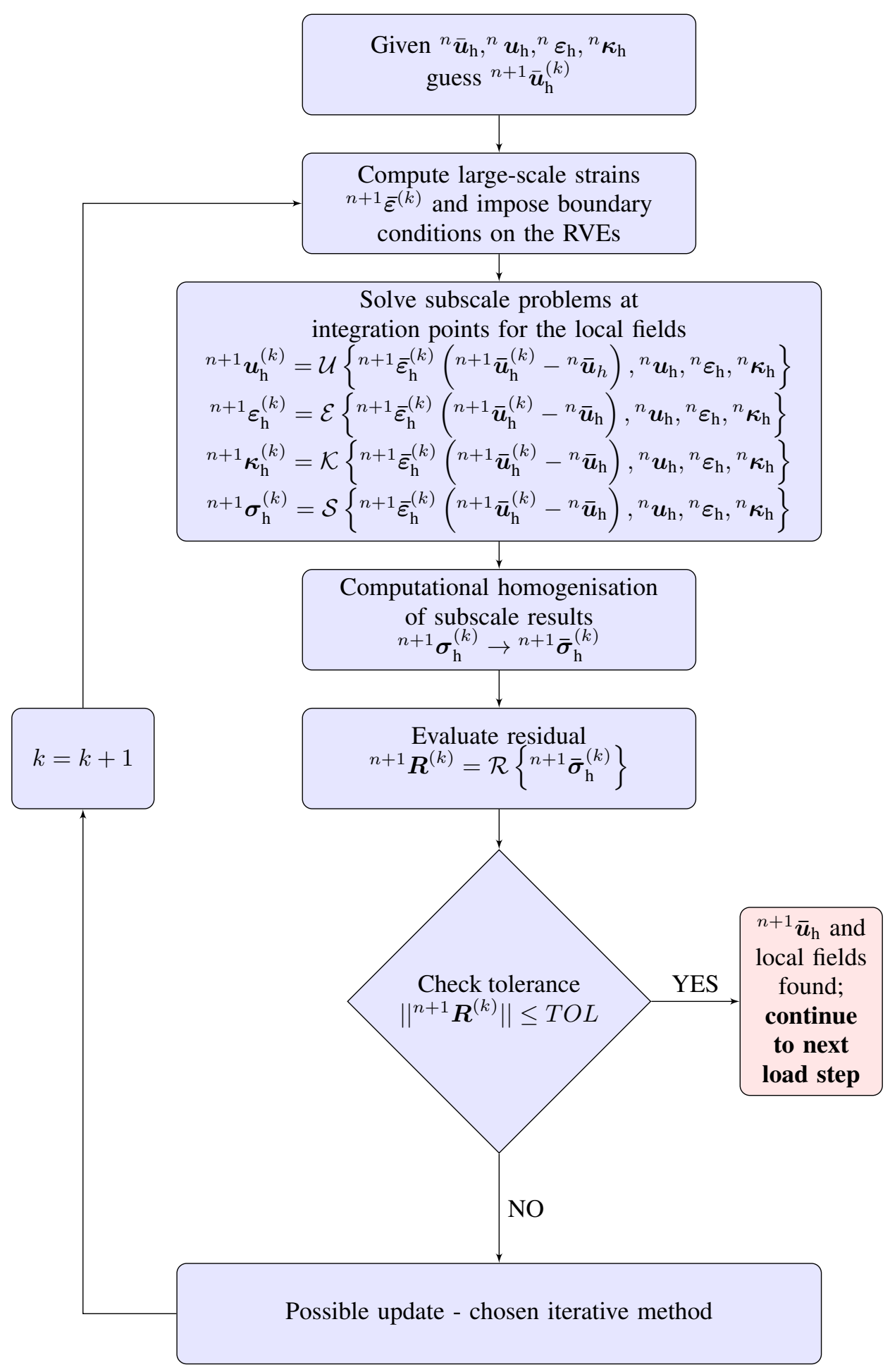

Figure 8: Flowchart of the nested finite element algorithm 
$1 \times 1$

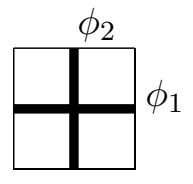

$L_{\square}=200 \mathrm{~mm}$
$2 \times 2$

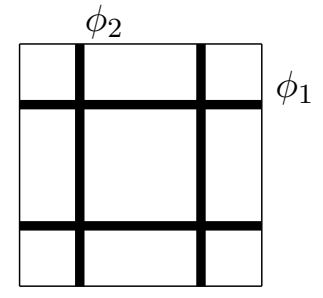

$L_{\square}=400 \mathrm{~mm}$
$3 \times 3$

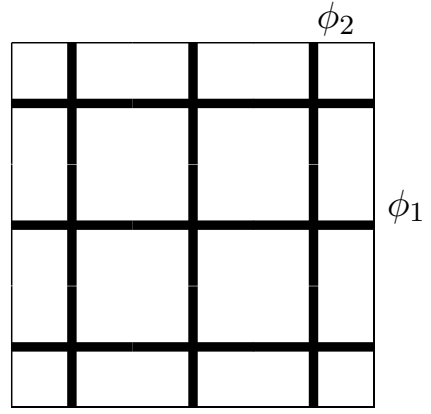

$L_{\square}=600 \mathrm{~mm}$

Figure 9: Three different RVEs used in the two-scale analysis.

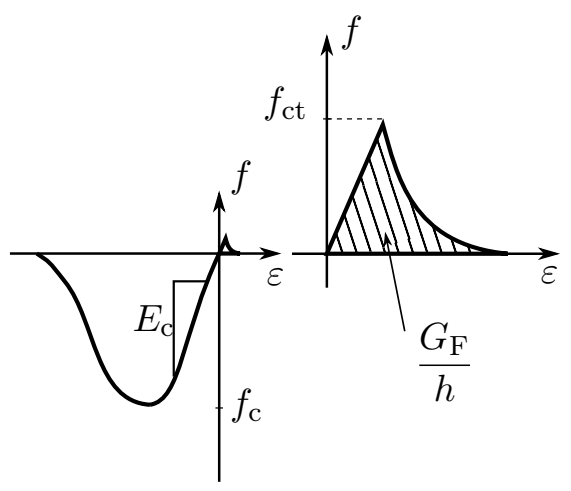

Figure 10: Stress-strain relation for concrete.

\begin{tabular}{ccc}
\hline & Description & Value \\
\hline$\rho_{\mathrm{c}}$ & density & $2400 \mathrm{~kg} / \mathrm{m}^{3}$ \\
$f_{\mathrm{c}}$ & compressive strength & $38 \mathrm{MPa}$ \\
$f_{\mathrm{ct}}$ & tensile strength & $2.9 \mathrm{MPa}$ \\
$G_{\mathrm{F}}$ & fracture energy & $140.5 \mathrm{~N} / \mathrm{m}$ \\
$E_{\mathrm{c}}$ & Young's modulus & $33.6 \mathrm{GPa}$ \\
$\nu_{\mathrm{c}}$ & Poisson's ratio & 0.2 \\
\hline
\end{tabular}

Table 1: Material parameters of concrete C30.

characteristics of the bond-slip interface were taken from the literature [4, 24] and are collected in Tables 1 to 3 . The constitutive behaviour of the materials and interfaces is also schematically depicted in Figures 10 to 12.

The reinforcement of type B500B was modelled as a von Mises elastoplastic material with strain hardening. Grade C30 concrete (according to the Model Code 2010 [4]) was considered for the bulk of the RVE. An isotropic continuum damage model, commonly referred to as the Mazars model [25, 26] was used to simulate its response. In the original Mazars model, the stress in tension asymptotically approaches its limit value and does not disappear completely. As a remedy, the damage evolution function in tension was modified accordingly, as detailed by Oliver et al. [27]:

$$
g_{t}(\kappa)= \begin{cases}0 & \text { if } \quad \kappa \leq \varepsilon_{0} \\ 1-\frac{\varepsilon_{0}}{\kappa} \exp \left(-\frac{\kappa-\varepsilon_{0}}{\varepsilon_{\mathrm{f}}}\right) & \text { if } \quad \kappa \geq \varepsilon_{0}\end{cases}
$$

where $\varepsilon_{0}=f_{\mathrm{ct}} / E_{\mathrm{c}}$ is the concrete strain at the onset of softening, and $\varepsilon_{\mathrm{f}}$ is a parameter, that depends among all on the fracture energy and the element size, cf. [27] for more details. The traction-separation law depicted in Figure 12 and specified in Table 3 was used to model the bond 


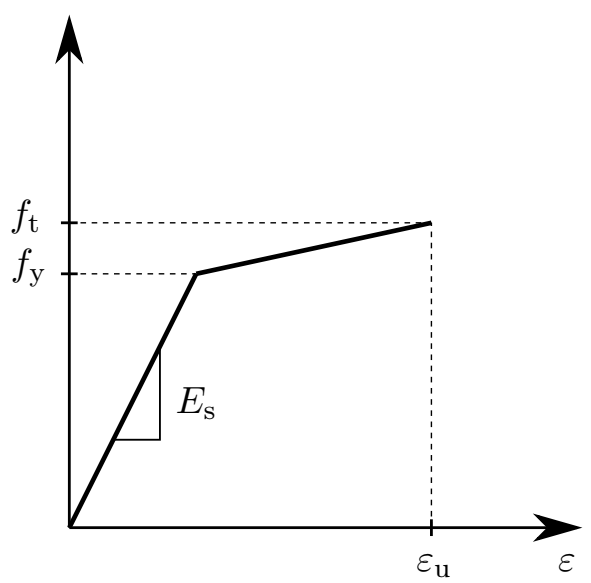

Figure 11: Stress-strain relation for reinforcing steel.

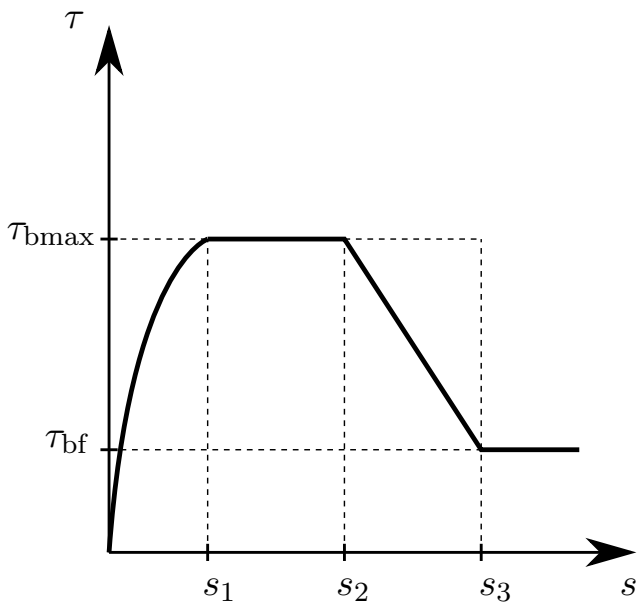

Figure 12: Bond stress-slip relation for steel- Table 3: Material parameters for concrete interface.

\begin{tabular}{ccc}
\hline & Description & Value \\
\hline$\rho_{\mathrm{s}}$ & density & $7850 \mathrm{~kg} / \mathrm{m}^{3}$ \\
$f_{\mathrm{y}}$ & yield strength & $500 \mathrm{MPa}$ \\
$f_{\mathrm{t}}$ & tensile strength & $540 \mathrm{MPa}$ \\
$E_{\mathrm{S}}$ & Young's modulus & $200 \mathrm{GPa}$ \\
$\nu_{\mathrm{s}}$ & Poisson's ratio & 0.3 \\
$\varepsilon_{\mathrm{u}}$ & strain at maximum & $5 \%$ \\
& force & \\
\hline
\end{tabular}

Table 2: Material parameters for reinforcing steel B500B

\begin{tabular}{ccc}
\hline & $\phi 8$ & $\phi 20$ \\
\hline$s_{1}$ & $1 \mathrm{~mm}$ & $1 \mathrm{~mm}$ \\
$s_{2}$ & $2 \mathrm{~mm}$ & $2 \mathrm{~mm}$ \\
$s_{3}$ & $4 \mathrm{~mm}$ & $6.5 \mathrm{~mm}$ \\
$\tau_{\text {bmax }}$ & $15.4 \mathrm{MPa}$ & $15.4 \mathrm{MPa}$ \\
$\tau_{\text {bf }}$ & $6.2 \mathrm{MPa}$ & $6.2 \mathrm{MPa}$ \\
\hline
\end{tabular}




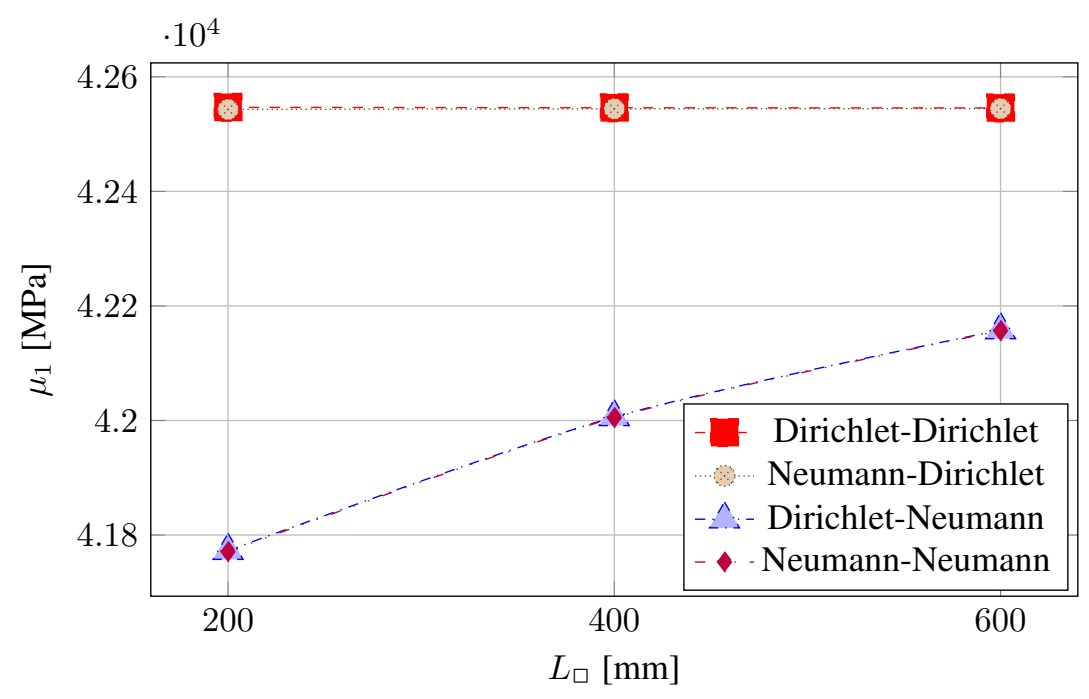

Figure 13: Influence of the RVE size on the largest eigenvalue, $\mu_{1}$, of the linear stiffness tensor.

characteristics.

According to the formulation presented in Section 2, the reinforcement bars were modelled as beams. In order to satisfy the constraint relation (equal displacement of steel and concrete perpendicular to the direction of the bar), the translational degrees of freedom of the steel were tied to the corresponding ones of the concrete along each bar. The element size along the bar conformed to the size of the concrete elements (quadrilaterals with bilinear displacement approximation). From the calibration of the modified Mazars model to match the desired strengths and to fit the elements of size $0.02 \mathrm{~m}$, the following set of parameters was found: $\varepsilon_{0}=8.63 \times 10^{-5}, \varepsilon_{\mathrm{f}}=0.0025, A_{\mathrm{c}}=2.6, B_{\mathrm{c}}=$ 800 For the steel/concrete interface, linear line interface elements were used. The thickness of the interface was set equal to the circumference of the pertinent reinforcing bar.

\subsection{Effective elastic stiffness}

In order to illustrate the effect of the unit cell size, $L_{\square}$, as well as the effect of different boundary conditions imposed on the RVE, the elastic stiffness tensor was computed. The largest eigenvalue of this tensor, $\mu_{1}$, has been plotted against the size of the RVE in Figure 13. The effect of $L_{\square}$ on the largest eigenvalue of the elastic stiffness tensor was most pronounced for the Dirichlet-Neumann and Neumann-Neumann boundary conditions, where a clear convergence pattern can be seen. For the Dirichlet-Dirichlet and Neumann-Dirichlet case, the quantity was also converging, but the difference between the two subsequent values was not substantial. Moreover, this example shows that the DD and NN conditions constitute the upper and lower bounds on the effective stiffness of the composite. ND and DN boundary conditions lie very near these bounds and could be used as an approximation of the upper/lower bound, if needed.

\subsection{Effective response in tension}

A homogenised stress-strain response of the RVEs is a valuable result, if the model should be further studied and validated. However, the strain localisation pattern and deformed shape of the RVEs provide also important information. To this end, tensile tests on the RVE were simulated. Uniaxial strain of $6 \times 10^{-3}$ was imposed on the RVEs via all discussed combinations of boundary conditions. 


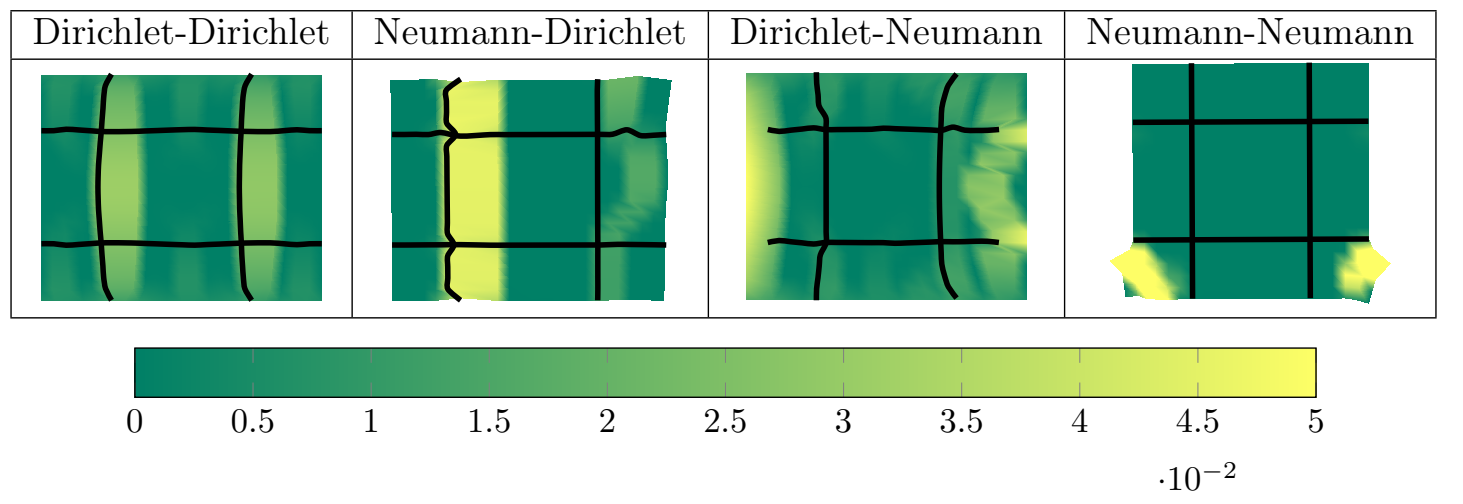

Figure 14: Principal strain $\varepsilon_{\text {I }}$ plotted on the deformed shape of the $2 \times 2$ RVEs. Magnification factor set to 40 for $\mathrm{DD}, \mathrm{ND}, \mathrm{DN}$ and 3 for $\mathrm{NN}$ boundary conditions.

The first principal strain, $\varepsilon_{\mathrm{I}}$, and the outline of the reinforcement bars (at the end of the tensile test) are plotted on the deformed shapes of the $2 \times 2$ RVEs in Figure 14 . The homogenised stresss-strain response is reproduced for all RVEs in Figure 15.

In the DD and ND cases, the strains tend to localise along the reinforcement bars. This is due to the fact that the thickness of the cross-section for the element rows along the reinforcement is reduced in order to conserve the total volume of the RVE. After initial cracking (loss of stiffness), the steel starts taking the stresses until it reaches yielding. This behaviour comports with the conventional understanding of reinforced concrete, and it is consistent for all analysed sizes of RVEs.

For the DN case, the strains tend to localise at the edges of the RVE (at later loading stages). Since it is only the concrete boundary that has a prescribed displacement, the steel bars are "free" to stay inside the RVE. This can cause large slip values, which in turn causes large bond stresses.

Finally, in the NN case both concrete and steel can deform freely at the boundary. As already observed in literature, the Neumann BCs are inaccurate in the fracturing continua [28, 29] and overall, predict very low stresses as only a piece of the microstructure can be deformed in order to fulfil the average strain constraint. This is in accordance in what we see in Figure 14 and Figure 15. In order to render the comparison of NN case with other boundary conditions possible, the magnification factor for the deformations has been reduced, and the actual principal strains lie outside the presented color scale.

In fact, this spurious localisation phenomenon is present also in ND case. It was observed that the iterative method used in the solution process has an influence on the final localisation pattern. While the Newton method with tangent stiffness yielded quick solution, the fracture in the RVE looks closer to the one present for the Neumann-Neumann boundary conditions, i.e. a certain piece of the RVE experiences high strain, while the rest stays in place. It might also vary between consecutive runs. Iterative methods using secant and elastic stiffness resulted in longer solution process, but the localisation pattern proved to be more stable and consistent between multiple runs (this was noticed especially for the modified Newton method using the elastic stiffness).

To summarise, after the preliminary study on the effective response of the RVEs, it can be expected that the DD and ND boundary condition constitute some measure of upper bound on the structural response, while the DN and NN boundary conditions constitute the lower bound. In addition, a softening response can be expected for structures modelled with DN and NN conditions at the subscale. 

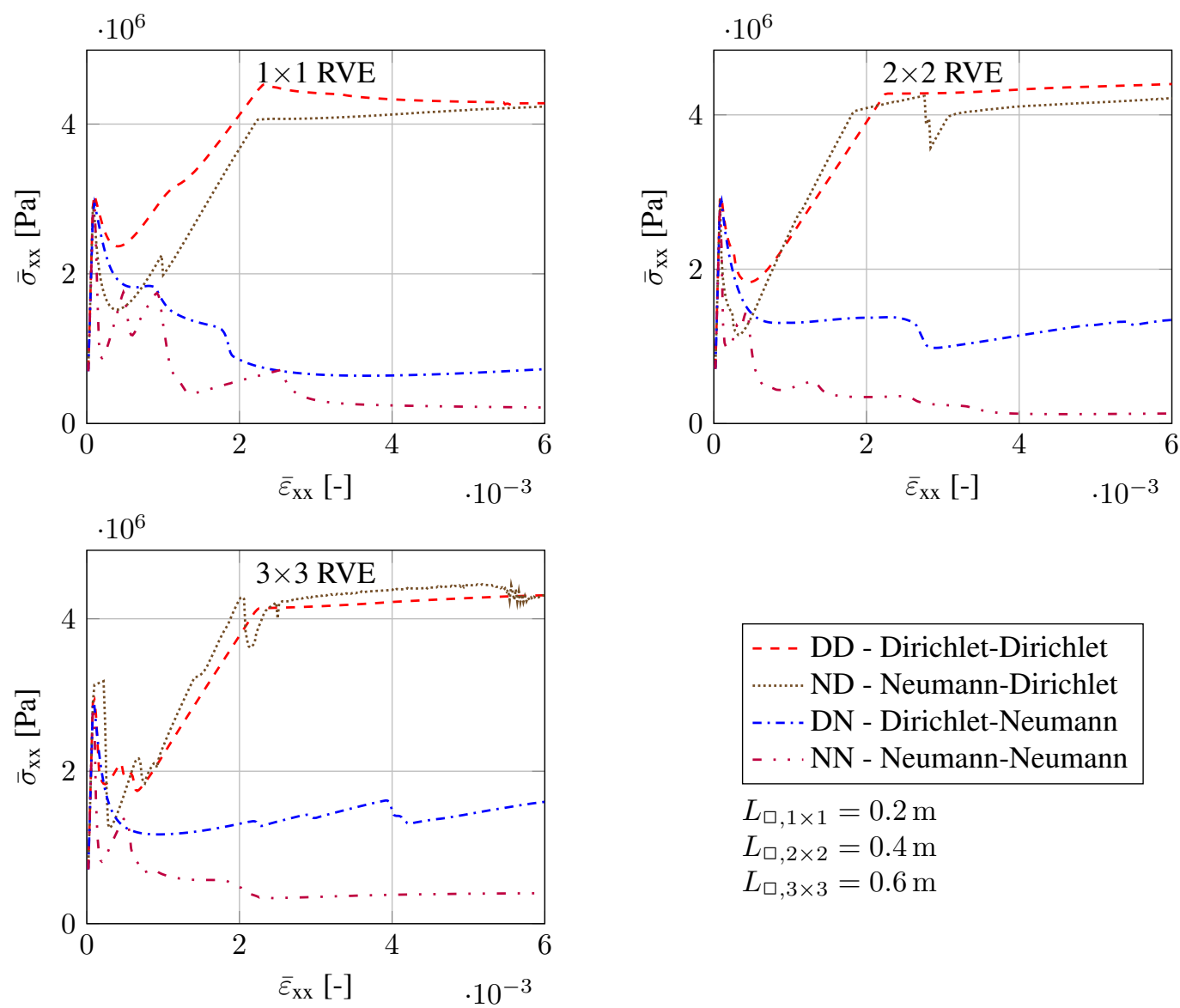

\section{- - - DD - Dirichlet-Dirichlet \\ .......... ND - Neumann-Dirichlet \\ -.-. DN - Dirichlet-Neumann \\ - ... NN - Neumann-Neumann}

$L_{\square, 1 \times 1}=0.2 \mathrm{~m}$

$L_{\square, 2 \times 2}=0.4 \mathrm{~m}$

$L_{\square, 3 \times 3}=0.6 \mathrm{~m}$

Figure 15: Homogenised stress-strain response of the RVEs under uniaxial tension. 


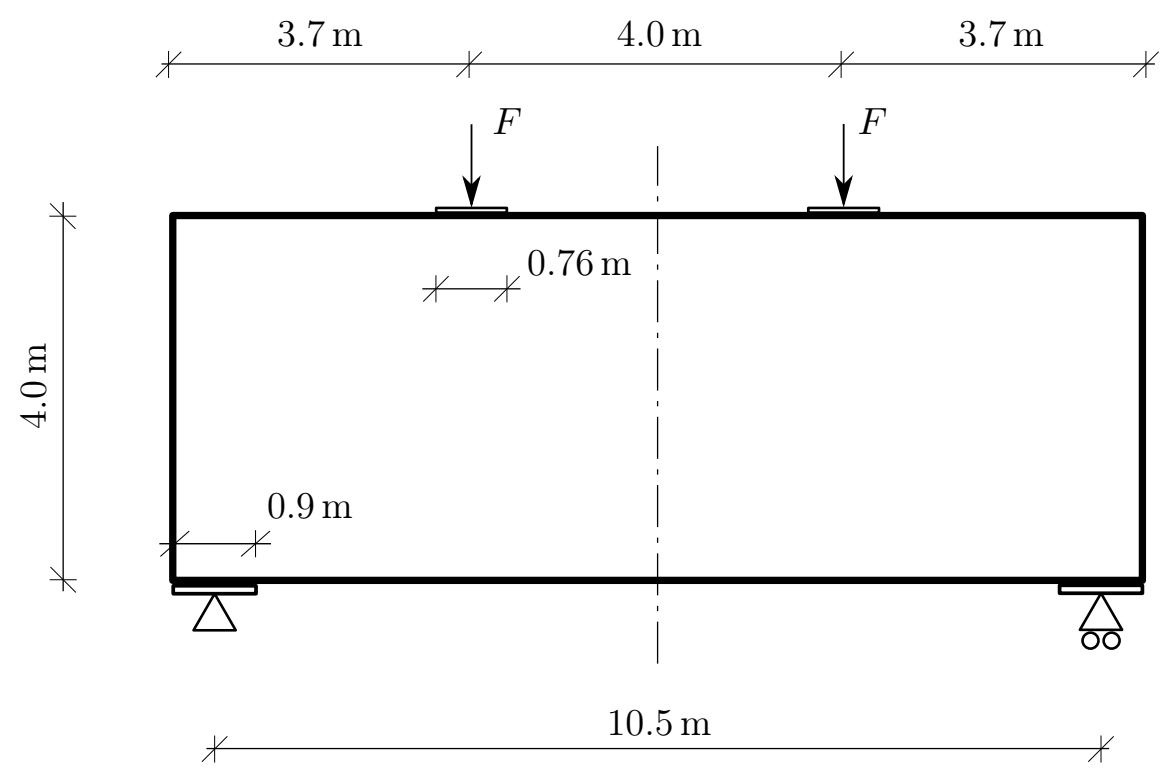

Figure 16: Deep beam of reinforced concrete loaded in four-point bending.

\section{Application example}

\subsection{Problem layout}

A simply supported deep beam of reinforced concrete, loaded in four-point bending, was chosen for the numerical study, see Figure 16. It was $10.5 \mathrm{~m}$ long, $4 \mathrm{~m}$ high and $0.2 \mathrm{~m}$ thick. Because the thickness was considerably smaller than the other dimensions, it was assumed that the structure could be successfully analysed as a two-dimensional solid in plane stress. The beam was reinforced with a uniform reinforcement grid in the entire structure.

The purpose of this example was to analyse the response of the deep beam in terms of deformation, crack pattern and crack widths. The first analysis used full resolution (also referred to in the literature as the Direct Numerical Simulation - DNS), i.e. formulation according to Section 2, whereas the subsequent analyses were based on two-scale formulation derived in Section 3 and the algorithm from Section 4. In both analysis types, the open source $\mathrm{C}++$ code OOFEM (www.oofem.org) [23] was used. It is of interest to know how these approaches compare to each other in terms of solution accuracy and computational time.

\subsection{Fully resolved model}

\subsubsection{Modelling choices}

For this analysis, the structure was modelled in full resolution, i.e. every reinforcement bar was modelled separately, cf. Figure 17. Moreover, in order to avoid singularities, the support and loading platens were resolved, and the nodes at these locations were tied accordingly to simulate rigid rotation and translation. Symmetry of the structure was used; hence, only half of the beam was modelled. As far as material models and element types are concerned, the same choices were made as those presented in Section 5. For the concrete elements along symmetry line, the crack band width was doubled, which corresponds to $\varepsilon_{\mathrm{f}}=0.00117$ in the Mazars model. 


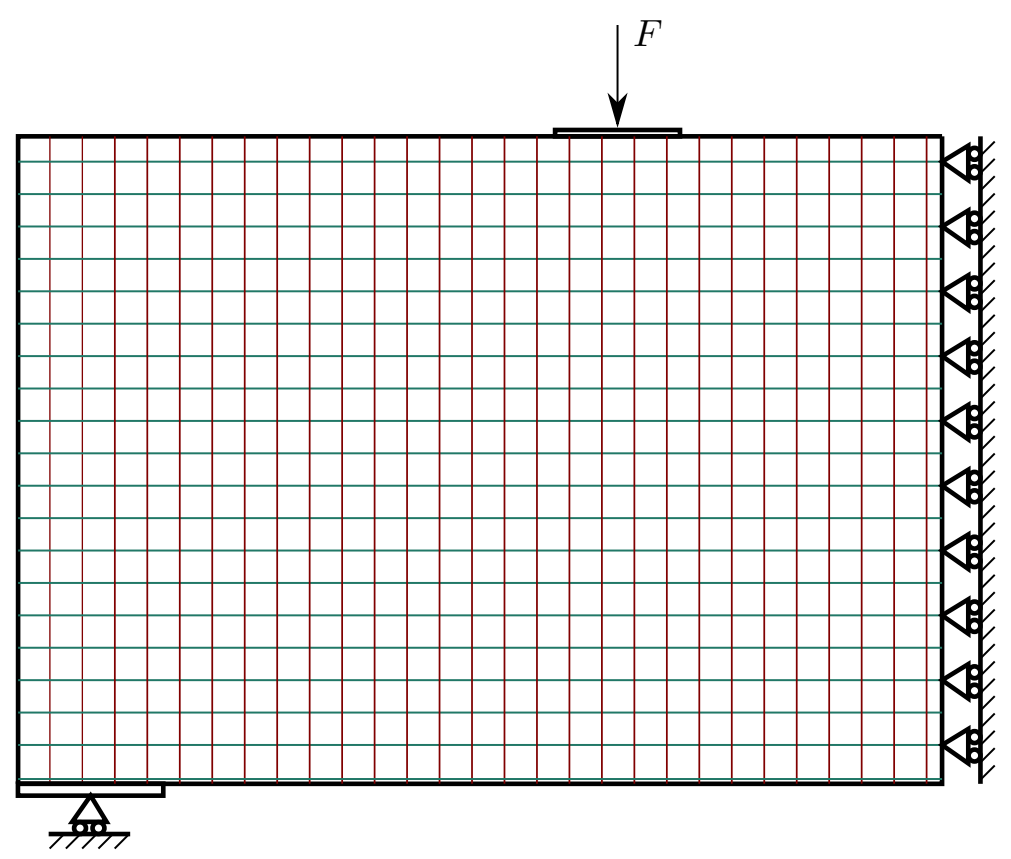

Figure 17: Fully resolved model of the deep beam.

\subsubsection{Analysis procedures}

A nonlinear analysis was performed under displacement control. The prescribed displacement under the loading plate was increased in 100 steps of $0.1 \mathrm{~mm}$. For equilibrium iterations, a Quasi-Newton method with secant stiffness was used with convergence criterion on the unbalanced forces.

\subsection{Two-scale model}

\subsubsection{Modelling choices}

As a first step in two-scale analysis, a resolution at the large- and subscale must be chosen. At large-scale, the deep beam was modelled with quadratic triangular elements (6 noded triangles with 4 Gauss quadrature points). Support and loading platens were emulated by tying appropriate degrees of freedom so that rigid rotation and translation at the support are accomplished. Because of symmetry, only half of the beam was modelled, with relevant degrees of freedom at the symmetry line locked, see Figure 18.

As for the RVEs, periodicity of the substructure was utilised. Moreover, since the reinforcement layout was uniform throughout the structure, one unit cell could be used to simulate the subscale behaviour of the material at all integration points. If the structure was not reinforced uniformly, it would still be possible to define different RVEs in different quadrature points. Material models and elements used in construction of the RVEs conform to those described in Section 5. In order to study the effect of unit cell size on the response of the model, the three different sized RVEs $(1 \times 1$, $2 \times 2,3 \times 3)$ were used in the analysis.

\subsubsection{Analysis procedures}

The $\mathrm{FE}^{2}$ analyses were run under displacement control, comprising 100 load steps of $0.1 \mathrm{~mm}$ deformation under the loading plate. The Modified Newton method was used for the equilibrium 


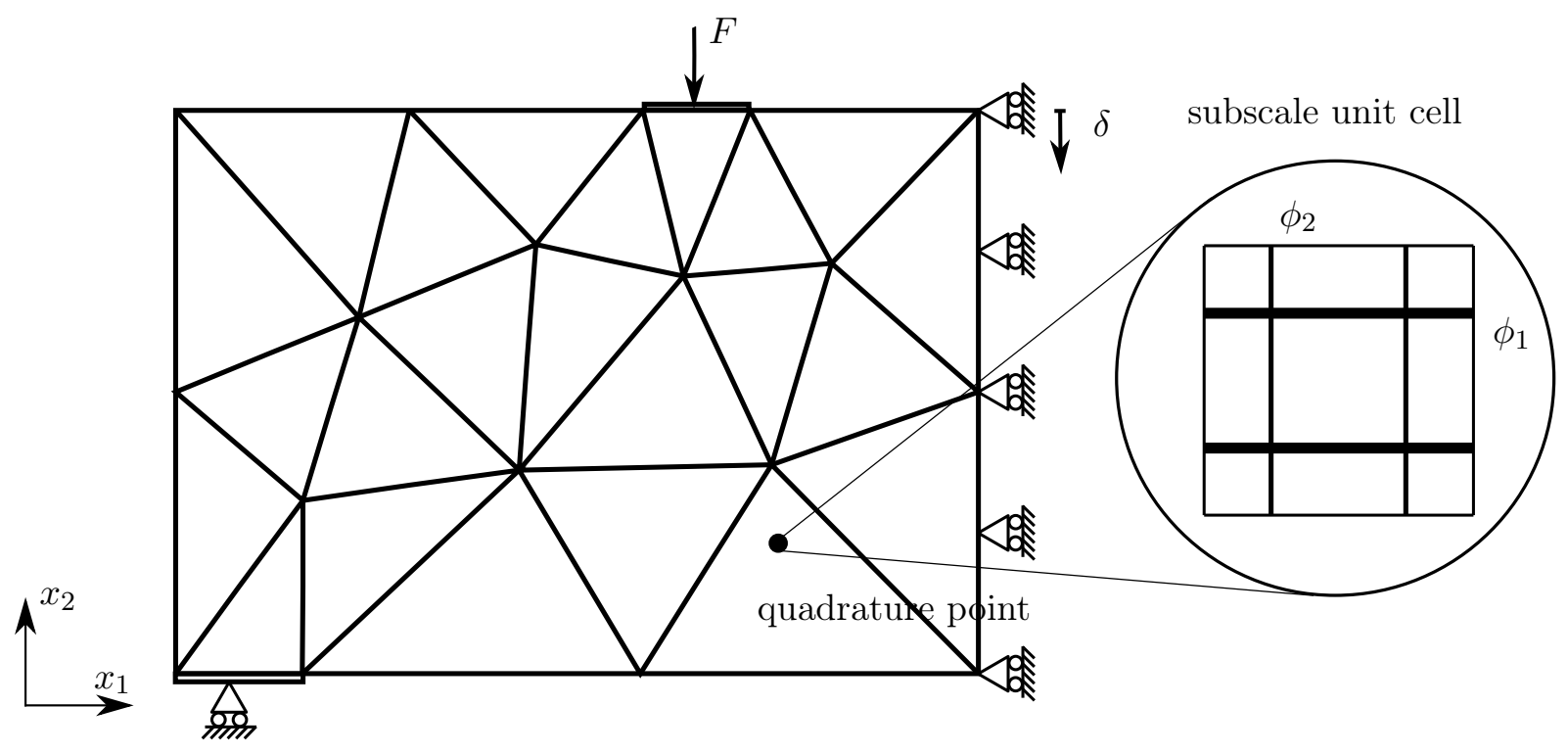

Figure 18: Two-scale model of the reinforced deep beam with a $2 \times 2$ subscale unit cell.

iteration at the large-scale, while at the subscale the Quasi-Newton secant method and Modified Newton method were used, as different combinations proved to be the most effective for different combinations of boundary conditions. The convergence criterion for both scales was set on the unbalanced forces. If convergence is not met within a preset number of iterations, the solution is accepted although being outside the specified tolerance. If this reoccurs frequently, the simulation is terminated.

\subsection{Computational results}

\subsubsection{Deformed shape}

The deformed shape of the structure is a conspicuous result, which can be used to verify the validity of the analysis. In Figure 19, the deformed shapes are compared between the fully resolved and two-scale analyses using $1 \times 1$ unit cells. The deformations at the locations of "macroscopic" nodes were extracted from the fully resolved solution in a postprocessing step. The undeformed shape was reproduced for reference. The results are from the last analysis step, i.e. when the deformation just beneath the loading platen equals $10 \mathrm{~mm}$.

Even though the two-scale analyses used the smallest RVE, a very good agreement between the deformed shapes can be observed for the Dirichlet-Dirichlet and Neumann-Dirichlet boundary conditions. The discrepancy between the deformed shapes obtained for Dirichlet-Neumann and Neumann-Neumann cases is attributed to the excessive softening exhibited by these two combinations of boundary conditions.

\subsubsection{Onset of localisation}

The initial phase of the general force-displacement response of the analysed structure is presented for the two-scale model for different RVEs and boundary conditions in Figure 20. It is possible to see stiffening effect in the analyses with DD and ND boundary conditions, and a clear softening behaviour of the structure when DN and NN boundary conditions were imposed on the RVEs. 


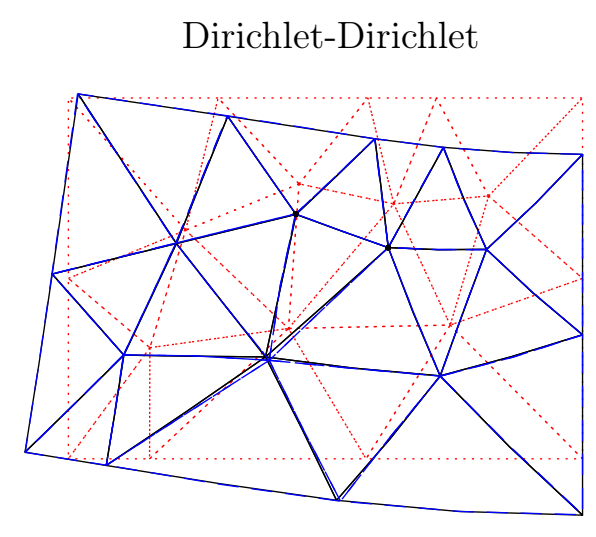

Neumann-Dirichlet

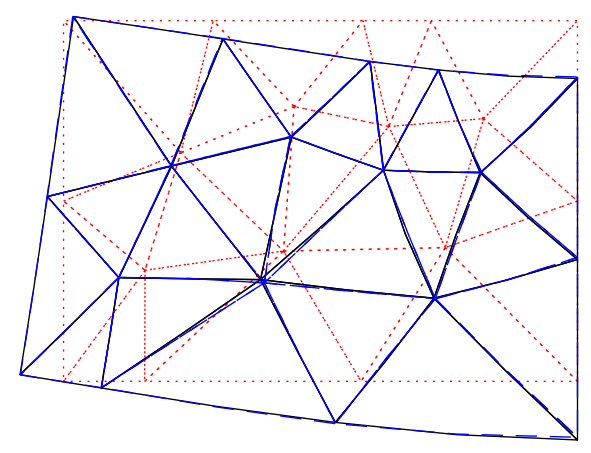

Dirichlet-Neumann

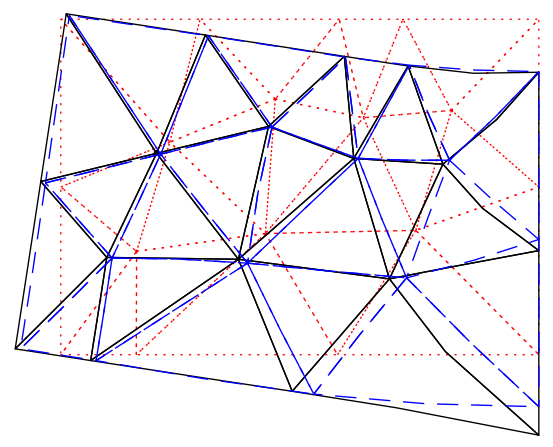

Neumann-Neumann

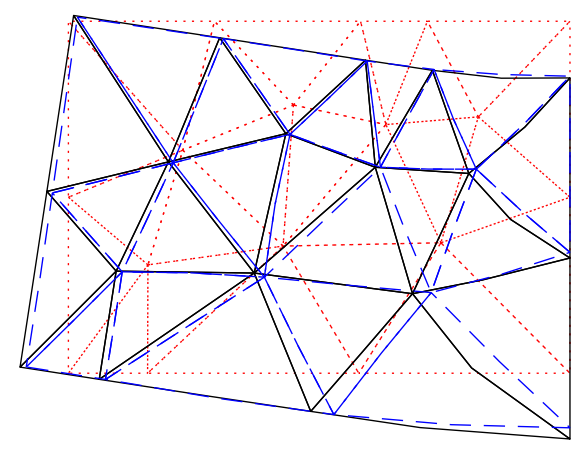

\section{Undeformed $\_\mathrm{FE}^{2}---$ Fully Resolved}

Figure 19: Deformed shapes of the deep beam at $10 \mathrm{~mm}$ deformation under loading plate (with magnification factor of 50). Two-scale analyses carried out with $1 \times 1$ RVEs. 

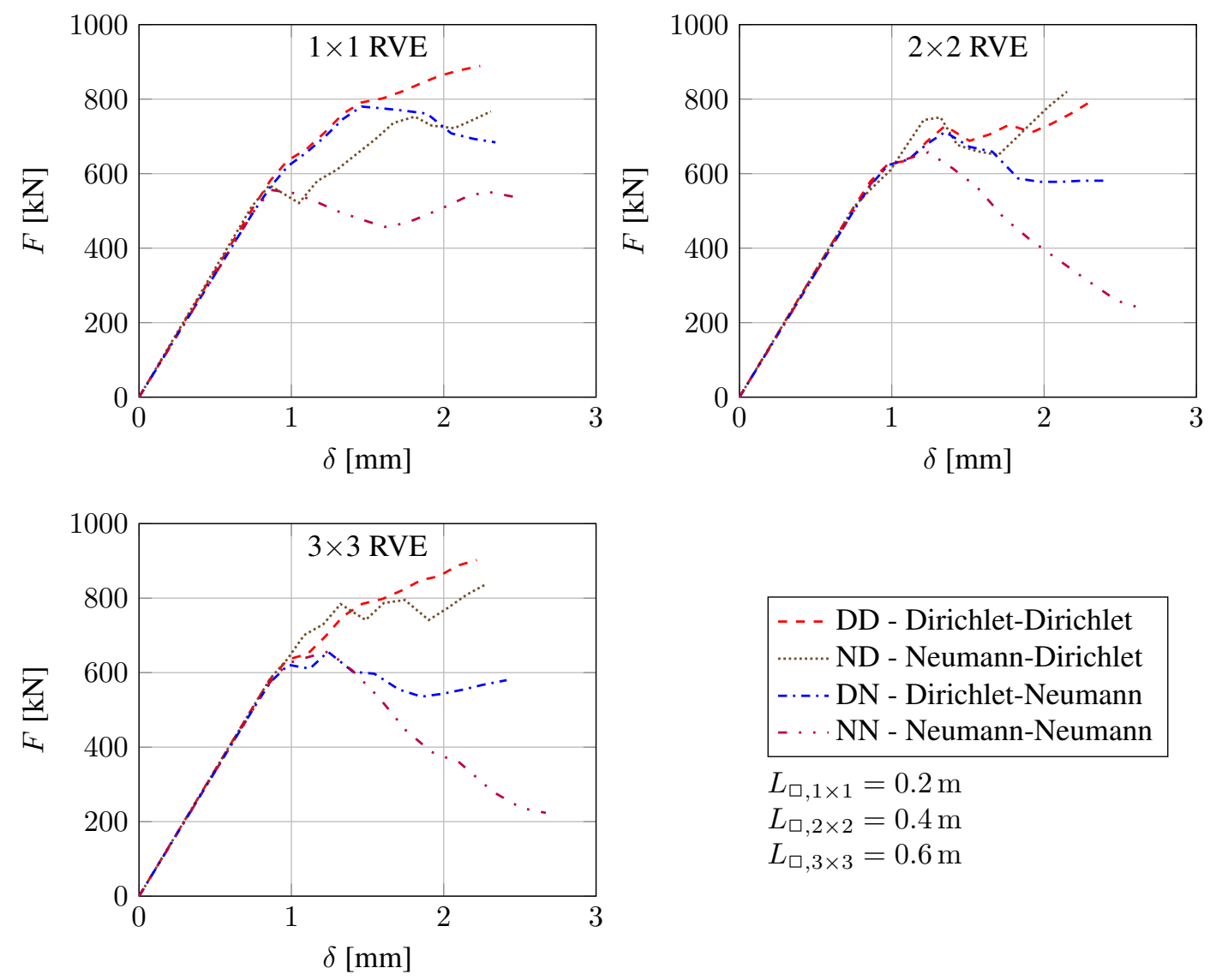

Figure 20: External load - mid-span deflection relation for two-scale analyses using different sizes of RVE with different boundary conditions, at the onset of strain localisation. External load, $F$, and mid-span defleciton, $\delta$, are defined in Figure 18. 

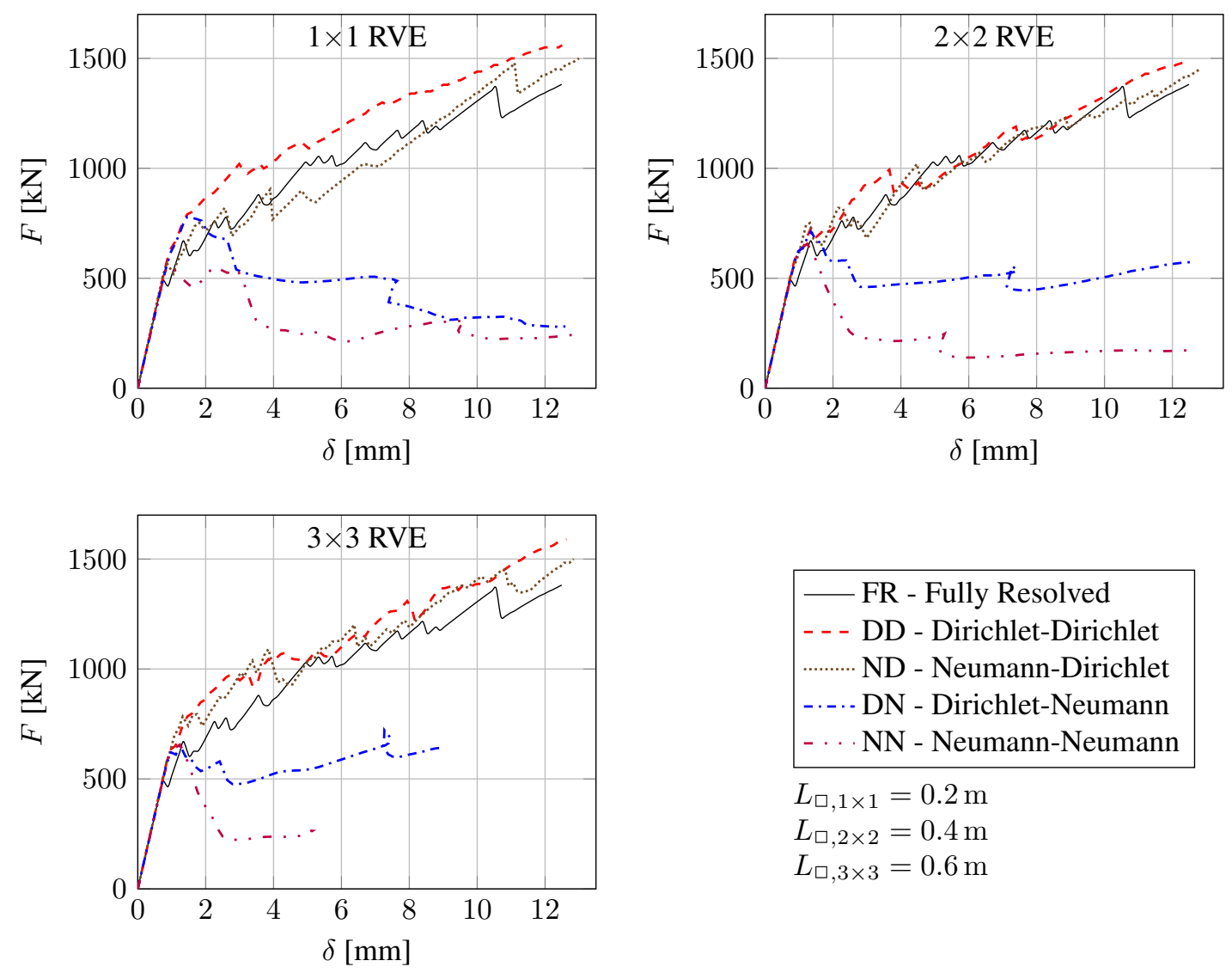

Figure 21: External load - mid-span deflection relations for two-scale analyses using different RVEs, compared to results from fully resolved analysis. External load, $F$, and mid-span defleciton, $\delta$, are defined in Figure 18.

\subsubsection{Force-deflection curves}

The relation between external load and the mid-span vertical deflection represents an important result describing the structural behaviour. Load-deflection curves for two-scale analyses with all possible subscale unit cells and all combinations of boundary conditions are depicted in Figure 21 along with the solution from the fully resolved analysis. From the figure, it is evident that the DD and ND boundary conditions provide a good approximation of the structural behaviour. In the case of Dirichlet-Dirichlet boundary conditions, the solution seemed to constitute an upper bound, i.e. the bigger the RVE, the closer it came to the fully resolved solution. On the other hand, a similar behaviour could not be observed for the case of ND boundary conditions - the results were close to the single-scale solution already for small RVEs. As previously alluded to, the DN and NN boundary conditions seemed to constitute a lower bound on the structural response of the deep beam, but the accuracy of these bounds was rather poor. Even though the results improve with increasing RVE size, the validity of the assumption of scale separation can only be investigated by comparing the results to single-scale analysis. Once the assumption has been introduced, the fact that we are oversampling the structure by using large RVEs does not introduce additional errors in the context of first order homogenisation. However, a significant increase in computational time is present for larger RVEs. 


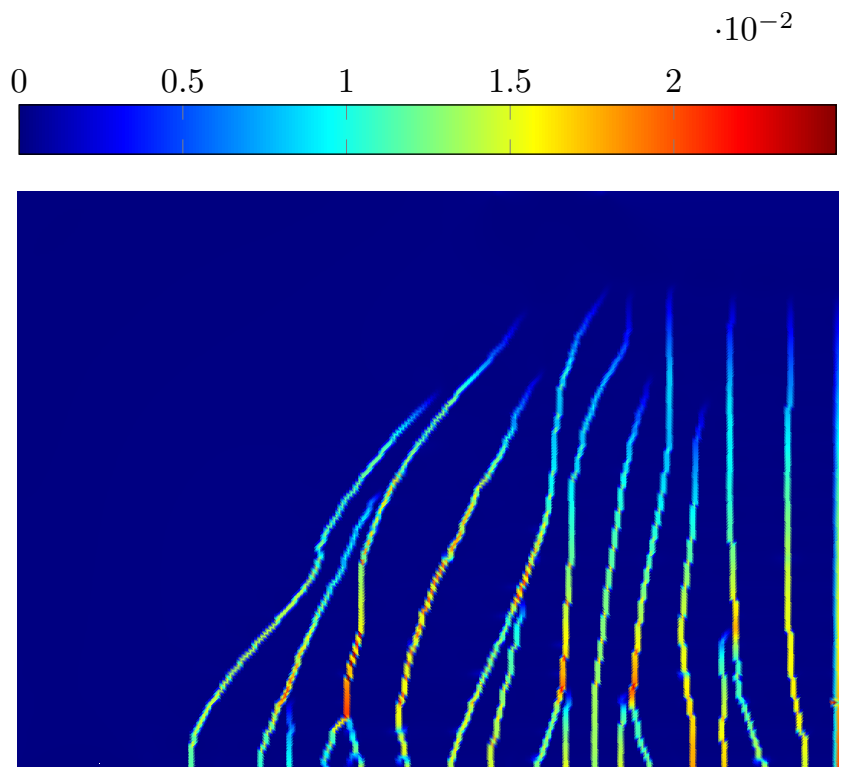

Figure 22: Principal strain $\varepsilon_{\mathrm{I}}$ for the fully resolved analysis at the last load step.

\subsubsection{Strain localisation}

For the fully resolved analysis, the strain localisation pattern can be obtained directly from the output. The principal strain, $\varepsilon_{\mathrm{I}}$, in the last analysis step is shown in Figure 22. For the two-scale analysis, strain localisation patterns can be recreated by combining results from both large-scale and subscale analyses. This was attempted in Figure 23, where the principal strain, $\varepsilon_{\mathrm{I}}$, and its direction were plotted for both the large-scale structure as well as for a few selected unit cells. The results are from the analyses with $2 \times 2$ unit cells with DD combination of boundary conditions. In general, the actual reinforcement layout in the vicinity of the Gauss point does not always tally with the location of the bars in the RVE. Having in mind the lack of objectivity of the subscale localisation patterns for the ND and NN boundary conditions (cf. Section 5.4), the reliability of the subscale results for the mentioned boundary conditions is dubious, and therefore it is deemed unrepresentative to reproduce them in separate figures. The subscale response of the two-scale analysis with DN boundary conditions is perhaps more stable, but since the lower bound is of low accuracy, it is not considered to be of great importance.

\section{Remark:}

In the fully resolved analysis, the cracks are not formed at locations of every vertical reinforcement bar, whereas for the RVEs they seem to form along each vertical rebar.

\subsubsection{Crack widths}

To predict the evolving crack width is of major practical importance, as it directly influences the durability of the structure and is therefore often limited in design codes. It is thus of interest to compare the largest crack widths obtained in the analyses. If the fully resolved analysis was not available, one would be limited to look at the large-scale integration point with largest strain, and extract the maximum crack width from the corresponding RVE output. Since the location of the largest crack was known from the fully resolved solution, the strain history could be extracted from the two-scale model at that location, and subsequent subscale analyses of an RVE were carried out 


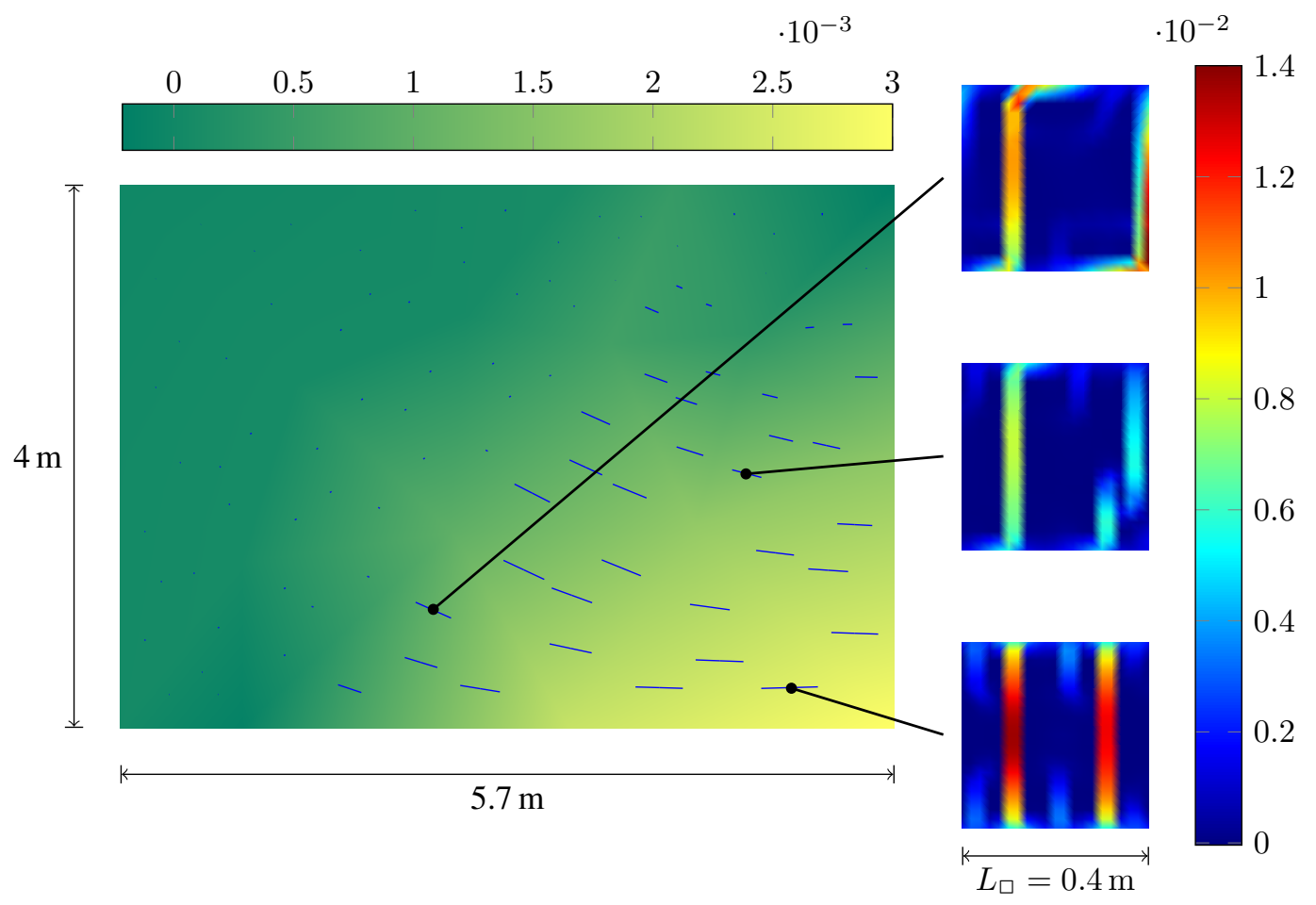

Figure 23: Magnitude and direction of the largest principal strain, $\varepsilon_{\mathrm{I}}$, in the deep beam for the $2 \times 2$ $\mathrm{DD} \mathrm{FE}^{2}$ analysis at the last load step.

using the prescribed strain history as input. Having in mind, that DD boundary conditions provided both an accurate bound on the structural response, as well as a stable subscale solution, it's natural to consider them for the subscale analysis, where crack widths are of importance.

The results for all sizes of unit cells are illustrated in Figure 24. The maximum crack widths obtained with the two-scale analyses were underestimated for all RVEs, although the larger the RVE, the larger the crack width computed from the subscale analysis. This is partly due to the nature of the Dirichlet-Dirichlet boundary conditions, and partly to the assumptions made in the beginning, namely the fact that the reinforcement slip is varying only at subscale. As shown in [28], Dirichlet boundary conditions on the boundary of the RVE might suppress the crack opening. Therefore, the crack widths might be expected to be underestimated. The authors believe, that allowing the reinforcement slip to vary in the large-scale model could alleviate this issue.

\subsubsection{Average strain}

The average strain over an arbitrary region can be computed for each two-scale analysis and compared with the fully resolved solution. This global measure also shows both the general behaviour of the structure and the accuracy of the two-scale analyses. The average strain $\bar{\varepsilon}_{\mathrm{xx}}$ was computed over the process zone of the deep beam at each deformation step and is reproduced in Figure 25. The average strain for DD and ND boundary conditions matched the average strain from the fully resolved solution well. Having in mind that the maximum crack width was underestimated when using the two-scale formulation, it can be inferred that there are more cracks (or that the cracks are more evenly distributed) than in the fully resolved analysis. The biggest discrepancies between the fully resolved and two-scale models could be observed for the NN and DN boundary conditions. For 

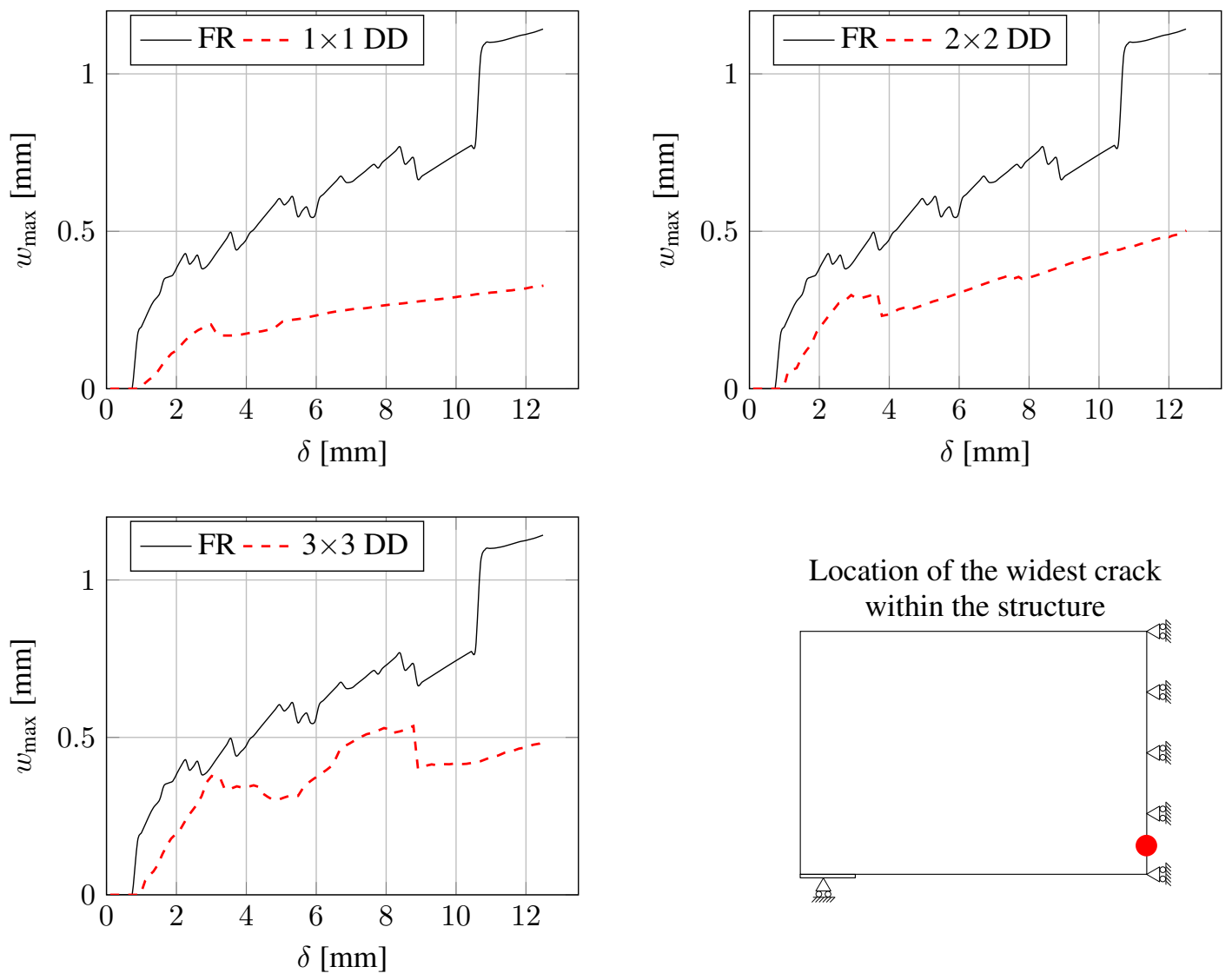

Location of the widest crack within the structure

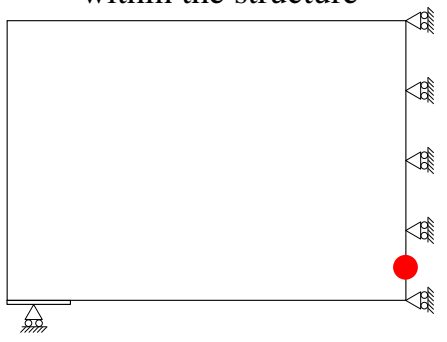

Figure 24: Maximum crack width, $w_{\max }$, versus mid-span deflection, $\delta$, for subscale analyses carried out at the depicted location using different sizes of the unit cell and DD boundary conditions. Compared with results from fully resolved analysis. 

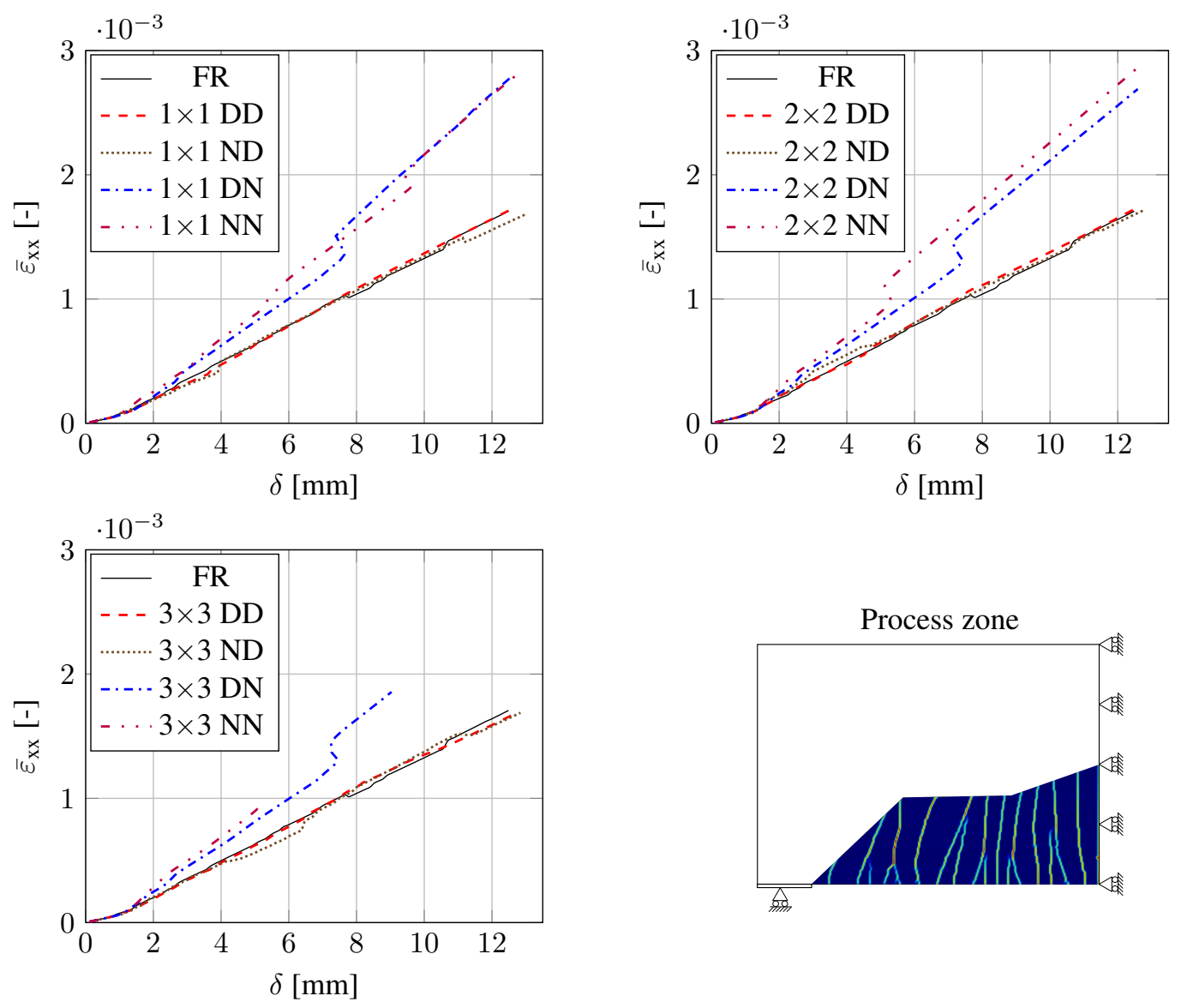

Figure 25: Average strain $\bar{\varepsilon}_{\mathrm{xx}}$ in the process zone (defined in the figure) of the deep beam for fully resolved and two-scale analyses using different sizes of subscale unit cell and boundary conditions.

those cases, the softening in the structure caused unnaturally large deformations in the lower right region of the beam (Figure 19), which in turn yielded too large strain on the average.

\subsubsection{Mesh sensitivity}

In order to investigate the influence of the large-scale mesh size on the results obtained with the two-scale model, the $\mathrm{FE}^{2}$ analysis was repeated on a number of meshes, schematically reproduced in Figure 26 along with their number of elements, $n_{\mathrm{el}}$. To shorten the computational time, the $1 \times 1 \mathrm{RVE}$ with Dirichlet-Dirichlet boundary conditions was used for the two-scale analyses. The main interest of this sensitivity study was to see if the load-deflection curve is affected by the mesh resolution, and also to determine the accuracy of the results obtained from the original mesh. The former query was addressed in Figure 27 (left), where it can be seen that there was little difference between the load-deflection curves for the considered meshes. For the latter question, the initial stiffness of the structure (initial slope of the force-deflection curve), $k$, was computed for all of the meshes and is compared to the fully resolved solution in Figure 27 (right). A clear convergence pattern can be seen, with the result approaching fully resolved solution for the larger meshes. For the original mesh, the relative difference between the two-scale and fully resolved solution was $3.26 \%$, while for the most refined mesh it was $0.56 \%$. 

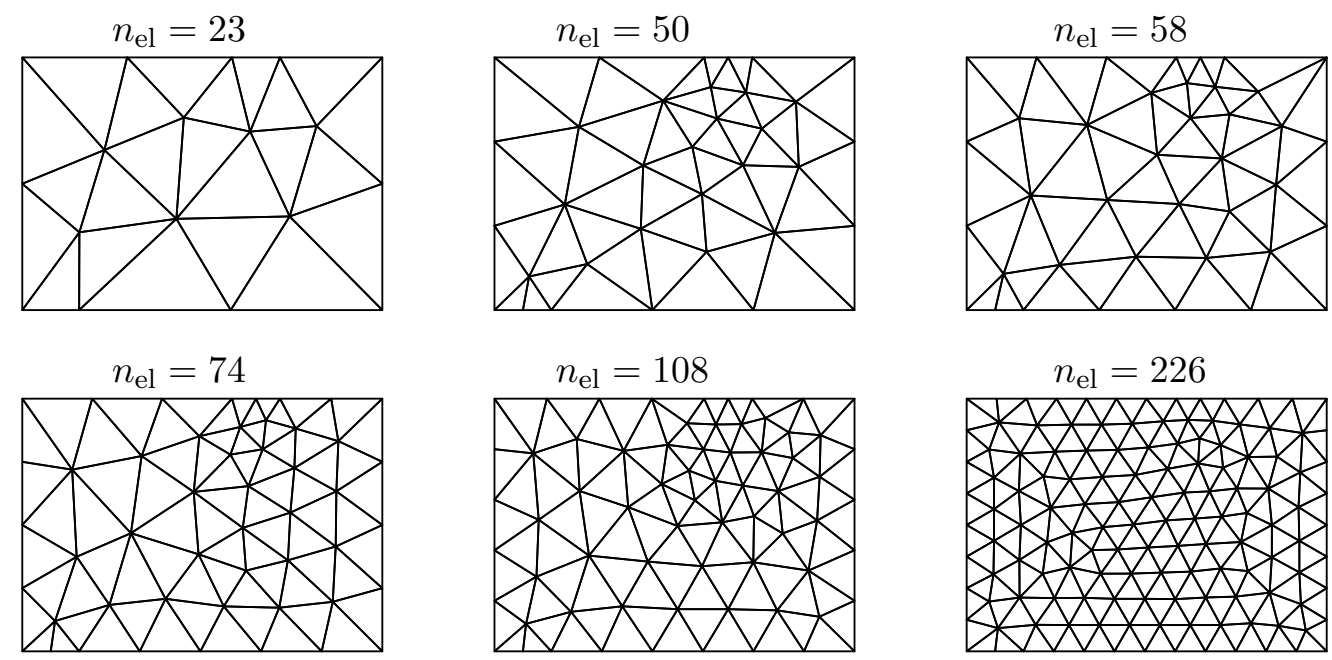

Figure 26: Different meshes used for the mesh sensitivity study.
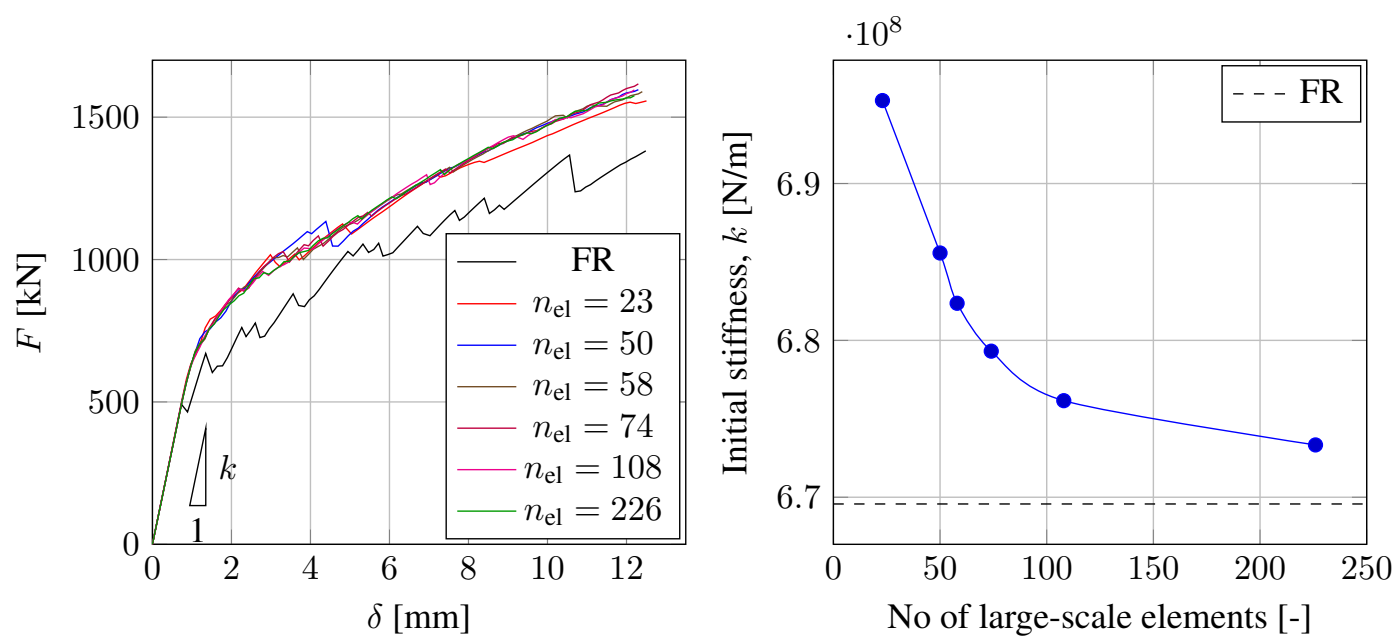

Figure 27: External load - mid-span deflection curves for different meshes (left). Initial stiffness for different meshes (right). 
Table 4: Computational times for the $\mathrm{FE}^{2}$ analyses using DD boundary conditions and the fully resolved analysis

\begin{tabular}{|c|c|c|c|}
\hline 1x1 DD & $2 \mathrm{x} 2 \mathrm{DD}$ & $3 \mathrm{x} 3 \mathrm{DD}$ & FR \\
\hline 1h:27m:37s & $8 \mathrm{~h}: 37 \mathrm{~m}: 34 \mathrm{~s}$ & $23 \mathrm{~h}: 35 \mathrm{~m}: 44 \mathrm{~s}^{1}$ & $15 \mathrm{~h}: 07 \mathrm{~m}: 04 \mathrm{~s}$ \\
\hline
\end{tabular}

\subsubsection{Computational cost}

One of the main reasons behind the development of multiscale methods in the recent years, is their potential to provide accurate results at a fraction of the single-scale computational time. In the case of the proposed two-scale model for reinforced concrete, several observations can be made. In terms of the size of the equation system, the fully resolved model contained 149278 free degrees of freedom. For solution, a parallel sparse direct solver (MUMPS), present in the PETSc family [30] was used. In comparison, the large-scale part of the two-scale model contained 103 degrees of freedom. The exact number of the degrees of freedom for the RVEs depends on the chosen boundary conditions, but at most, the $1 \times 1,2 \times 2$ and $3 \times 3$ RVEs contained 288, 1060 and 2308 degrees of freedom, respectively. A direct solver from the PETSc family was used to solve the large-scale problem, as well as the subscale problems with Dirichlet-Dirichlet boundary conditions. Unfortunately, it was not possible to handle the DN, ND, and NN boundary conditions directly with the same PETSc solver, and it was necessary to shift to OOFEM's default direct solver, which resulted in long computation times. Therefore, a direct comparison of computational times for the DN, ND and NN boundary conditions at the subscale is deemed biased. The times analyses with DD boundary conditions are presented in Table 4.

It is noteworthy, that while a secant stiffness was used in equilibrium iterations for the two smallest RVEs, the largest RVE required a change to a Modified Newton method using the linear stiffness in iterations, which further prolonged the computational time. By looking at the computational times, it can be said that the two-scale analyses with Dirichlet-Dirichlet boundary conditions provide an attractive alternative to the single-scale analysis, especially if the RVE used is small.

\section{Conclusions and outlook}

In this paper, a two-scale model of reinforced concrete considering the bond-slip relation between steel and concrete was derived. First-order homogenisation was employed, i.e. standard linear variation of the unknown displacements fields within the RVE. It is noteworthy that the homogenisation was selective in the sense that it was only the concrete displacement that possessed an independent macroscopic component. Moreover, the variation of reinforcement slip was considered only locally, i.e. at the subscale. Both Dirichlet and Neumann boundary conditions were imposed on the concrete solid and reinforcement of the RVE. Hence, a total of four alternative boundary conditions were studied: Dirichlet-Dirichlet(DD), Dirichlet-Neumann(DN), Neumann-Dirichlet(ND) and Neumann-Neumann(NN). The DD and NN boundary conditions provided upper and lower bounds on the effective stiffness of the composite material. In terms of structural response, excessive softening could be observed for the two-scale analyses with DN and NN boundary conditions on the RVEs; this signifies the potential infeasibility of these types of boundary conditions in modelling of reinforced concrete structures. Moreover, the results obtained with ND and NN results lacked objectivity with respect to strain localisation patterns and the usefulness of the produced subscale results remains uncertain. The model for both DD and ND conditions yielded promising results in

\footnotetext{
${ }^{1}$ Required change to Modified Newton method for subscale iterations.
} 
terms of the structural behaviour: the deformed shape, load-deflection relation and average strains agreed well between two- and single-scale analyses of the studied structure even for small sizes of the representative volume elements. Even though the maximum crack widths were underestimated, the mentioned Dirichlet-Dirichlet combination provided an approximate upper bound on the structural response in the considered problem.

For the future studies, the slip should be considered as a global variable, in which case some measure of average reinforcement slip over an area would be homogenised in the $\mathrm{FE}^{2}$ setting. Additionally, the heterogeneous composition of the concrete could be incorporated at the RVE level, thus creating a mesoscopic structure comprising the aggregates, cement paste, interfacial transition zone, and the reinforcement. A yet further step is to extend the existing framework from solid elements to shell elements, by which bending can be described and large (structurally) reinforced concrete structures, such as e.g. bridges, could be analysed in a multiscale manner.

\section{Acknowledgements}

This research was financially supported by The Swedish Research Council (Vetenskapsrådet) grant no. 621-2014-5168, which is gratefully acknowledged. The computations were performed on resources at the Chalmers Centre for Computational Science and Engineering (C3SE) provided by the Swedish National Infrastructure for Computing (SNIC).

\section{Appendix A Hill-Mandel macrohomogeneity condition}

We shall now show that the formulations pertinent to the DD, DN, ND and NN boundary conditions in Equations (77)-(80), (82)-(85), (87)-(91) and (92)-(96), respectively, satisfy the so-called macrohomogeneity condition. This condition, also denoted the Hill-Mandel condition, ensures equivalence of virtual work on the two scales. If there exists an underlying energy for the problem, the macrohomogeneity thus guarantees energy equivalence across the scales, cf. Hill [31].

\section{A.1 Canonical form of the RVE problem}

We first claim that all formulations (DD, DN, ND, NN) can be stated in the canonical form as follows: Find $\boldsymbol{u}_{\mathrm{c}}, u_{\mathrm{s}, 1}, u_{\mathrm{s}, \perp}, \lambda \in \mathbb{U}_{\square, \mathrm{c}}^{\star} \times \mathbb{U}_{\square, \mathrm{s}, \mathrm{l}}^{\star} \times \mathbb{U}_{\square, \mathrm{s}, \perp}^{\star} \times \mathbb{L}_{\square}$ and $\hat{\boldsymbol{\sigma}}_{\mathrm{c}} \in \mathbb{R}^{2 \times 2}$ such that

$$
\begin{array}{rlrl}
a_{\square, \mathrm{c}}\left(\boldsymbol{u}_{\mathrm{c}} ; \delta \boldsymbol{u}_{\mathrm{c}}\right)-b_{\square}\left(u_{\mathrm{s}, 1}-\boldsymbol{e}_{\mathrm{l}} \cdot \boldsymbol{u}_{\mathrm{c}} ; \boldsymbol{e}_{\mathrm{l}} \cdot \delta \boldsymbol{u}_{\mathrm{c}}\right) & & \\
-c_{\square}\left(\lambda ; \boldsymbol{e}_{\perp} \cdot \delta \boldsymbol{u}_{\mathrm{c}}\right)-d_{\square}\left(\hat{\boldsymbol{\sigma}}_{\mathrm{c}} ; \delta \boldsymbol{u}_{\mathrm{c}}\right) & =0 & & \\
a_{\square, 1}\left(u_{\mathrm{s}, 1}, u_{\mathrm{s}, \perp} ; \delta u_{\mathrm{s}, 1}\right)+b_{\square}\left(u_{\mathrm{s}, 1}-\boldsymbol{e}_{\mathrm{l}} \cdot \boldsymbol{u}_{\mathrm{c}} ; \delta u_{\mathrm{s}, 1}\right) & =0 & & \forall \delta u_{\mathrm{s}, \mathrm{c}} \in \mathbb{U}_{\square, \mathrm{s}, 1}^{0}, \\
a_{\square, \mathrm{b}}\left(u_{\mathrm{s}, 1}, u_{\mathrm{s}, \perp} ; \delta u_{\mathrm{s}, \perp}\right)+c_{\square}\left(\lambda ; \delta u_{\mathrm{s}, \perp}\right) & =0 & \forall \delta u_{\mathrm{s}, \perp} \in \mathbb{U}_{\square, \mathrm{s}, \perp}^{0}, \\
c_{\square}\left(\delta \lambda ; u_{\mathrm{s}, \perp}-\boldsymbol{e}_{\perp} \cdot \boldsymbol{u}_{\mathrm{c}}\right) & =0 & \forall \delta \lambda \in \mathbb{L}_{\square}, \\
-d_{\square}\left(\delta \hat{\boldsymbol{\sigma}}_{\mathrm{c}} ; \boldsymbol{u}_{\mathrm{c}}\right) & =-\delta \hat{\boldsymbol{\sigma}}_{\mathrm{c}}: \overline{\boldsymbol{\varepsilon}} & \forall \delta \hat{\boldsymbol{\sigma}}_{\mathrm{c}} \in \mathbb{R}^{2 \times 2},
\end{array}
$$

where the RVE-forms $a_{\square, \mathrm{c}}(\bullet ; \bullet), b_{\square}(\bullet ; \bullet), c_{\square}(\bullet ; \bullet), a_{\square, \mathrm{l}}(\bullet ; \bullet)$ and $a_{\square, \mathrm{b}}(\bullet ; \bullet)$ were introduced in Section 3.1 , and

$$
d_{\square}(\overline{\boldsymbol{\tau}}, \boldsymbol{w})=\frac{1}{\left|\Omega_{\square}\right|} \int_{\Omega_{\square, \mathrm{c}}}[\boldsymbol{w} \otimes \nabla] \mathrm{d} \Omega: \overline{\boldsymbol{\tau}}, \quad \overline{\boldsymbol{\tau}} \in \mathbb{R}^{2 \times 2} .
$$

The space $\mathbb{L}_{\square}$ was defined in Section 3.3, whereas remaining sets can be chosen in order to retain the pertinent formulation. 
The DD and DN formulations in (77)-(80) and (82)-(85), respectively, are retained by setting

$$
\mathbb{U}_{\square, \mathrm{c}}^{\star}=\mathbb{U}_{\square, \mathrm{c}}^{D}=\left\{\boldsymbol{v}(\boldsymbol{x}) \in \mathbb{U}_{\square, \mathrm{c}}: \boldsymbol{v}=\hat{\boldsymbol{\varepsilon}} \cdot[\boldsymbol{x}-\overline{\boldsymbol{x}}] \text { on } \Gamma_{\square} \text { for some } \hat{\boldsymbol{\varepsilon}} \in \mathbb{R}^{2 \times 2}:\right\},
$$

where $\mathbb{U}_{\square, \mathrm{c}}$ was defined in Section 3.3. Defining the test space in (A.1) as $\mathbb{U}_{\square, \mathrm{c}}^{0}=\mathbb{U}_{\square, \mathrm{c}}^{D}$ whilst employing the decomposition $\mathbb{U}_{\square, \mathrm{c}}^{D} \ni \quad \delta \boldsymbol{u}_{\mathrm{c}}=\delta \hat{\boldsymbol{\varepsilon}} \cdot[\boldsymbol{x}-\overline{\boldsymbol{x}}]+\delta \boldsymbol{u}_{\mathrm{c}}^{\mathrm{s}}$, results in (77) and (82) for $\delta \boldsymbol{u}_{\mathrm{c}}^{\mathrm{s}} \in \mathbb{U}_{\square, \mathrm{c}}^{D}(\mathbf{0})$. The evaluation for $\delta \boldsymbol{u}_{\mathrm{c}}=\delta \hat{\boldsymbol{\varepsilon}} \cdot[\boldsymbol{x}-\overline{\boldsymbol{x}}]$ simply defines the value of $\hat{\boldsymbol{\sigma}}_{\mathrm{c}}$. ND and NN formulations are obtained by setting $\mathbb{U}_{\square, \mathrm{c}}^{\star}=\mathbb{U}_{\square, \mathrm{c}}^{0}=\mathbb{U}_{\square, \mathrm{c}}^{\mathrm{N}}$, as defined in Section 3.3. Equations (A.1) and (A.5) clearly represent Equations (87)-(88) and (92)-(93). Regarding the reinforcement, DD and ND boundary conditions are obtained by setting

$$
\begin{array}{ll}
\mathbb{U}_{\square, \mathrm{s}, \mathrm{l}}^{\star}=\mathbb{U}_{\square, \mathrm{s}, \mathrm{l}}^{D}(\bar{\varepsilon}), & \mathbb{U}_{\square, \mathrm{s}, \perp}^{\star}=\mathbb{U}_{\square, \mathrm{s}, \perp}^{D}(\bar{\varepsilon}), \\
\mathbb{U}_{\square, \mathrm{s}, \mathrm{l}}^{0}=\mathbb{U}_{\square, \mathrm{s}, \mathrm{l}}^{D}(\mathbf{0}), & \mathbb{U}_{\square, \mathrm{s}, \perp}^{0}=\mathbb{U}_{\square, \mathrm{s}, \perp}^{D}(\mathbf{0}) .
\end{array}
$$

Finally, DN and NN boundary conditions are obtained by setting

$$
\mathbb{U}_{\square, \mathrm{s}, \mathrm{l}}^{\star}=\mathbb{U}_{\square, \mathrm{s}, \mathrm{l}}^{0}=\mathbb{U}_{\square, \mathrm{s}, \mathrm{l}}, \quad \mathbb{U}_{\square, \mathrm{s}, \perp}^{\star}=\mathbb{U}_{\square, \mathrm{s}, \perp}^{0}=\mathbb{U}_{\square, \mathrm{s}, \perp} .
$$

\section{A.2 Fulfilment of the macrohomogeneity condition}

In order to show that the macrohomogeneity condition is, indeed, satisfied, we consider the solutions $\left(\boldsymbol{u}_{\mathrm{c}}, u_{\mathrm{s}, 1}, u_{\mathrm{s}, \perp}, \lambda, \hat{\boldsymbol{\sigma}}_{\mathrm{c}}\right)$ and arbitrary virtual displacements $\left(\mathrm{d} \boldsymbol{u}_{\mathrm{c}}, \mathrm{d} u_{\mathrm{s}, 1}, \mathrm{~d} u_{\mathrm{s}, \perp}\right)$ brought on by the macroscopic update $\mathrm{d} \overline{\boldsymbol{\varepsilon}}=[\mathrm{d} \overline{\boldsymbol{u}} \otimes \boldsymbol{\nabla}]^{\mathrm{sym}}$. Here, from the reduced VMS ansatz together with Equations (65)-(67), it follows that

$$
\begin{aligned}
\mathrm{d} \boldsymbol{u}_{\mathrm{c}} & =\mathrm{d} \boldsymbol{u}_{\mathrm{c}}^{M}+\mathrm{d} \boldsymbol{u}_{\mathrm{c}}^{s}=\mathrm{d} \overline{\boldsymbol{\varepsilon}} \cdot[\boldsymbol{x}-\overline{\boldsymbol{x}}]+\mathrm{d} \boldsymbol{u}_{\mathrm{c}}^{s}, \\
\mathrm{~d} u_{\mathrm{s}, 1} & =\mathrm{d} u_{\mathrm{s}, 1}^{M}+\mathrm{d} u_{\mathrm{s}, 1}^{s}=\boldsymbol{e}_{\mathrm{l}} \cdot \mathrm{d} \overline{\boldsymbol{\varepsilon}} \cdot[\boldsymbol{x}-\overline{\boldsymbol{x}}]+\mathrm{d} u_{\mathrm{s}, 1}^{s}, \\
\mathrm{~d} u_{\mathrm{s}, \perp} & =\mathrm{d} u_{\mathrm{s}, \perp}^{M}+\mathrm{d} u_{\mathrm{s}, \perp}^{s}=\boldsymbol{e}_{\perp} \cdot \mathrm{d} \overline{\boldsymbol{\varepsilon}} \cdot[\boldsymbol{x}-\overline{\boldsymbol{x}}]+\mathrm{d} u_{\mathrm{s}, \perp}^{s},
\end{aligned}
$$

whereby $\mathrm{d} \boldsymbol{u}_{\mathrm{c}}^{s}, \mathrm{~d} u_{\mathrm{s}, 1}^{s}, \mathrm{~d} u_{\mathrm{s}, \perp}^{s} \in \mathbb{U}_{\square, \mathrm{c}}^{0} \times \mathbb{U}_{\square, \mathrm{s}, 1}^{0} \times \mathbb{U}_{\square, \mathrm{s}, \perp}^{0}$. The Hill-Mandel macrohomogeneity condition can now be stated in terms of requiring equivalence of virtual work

$$
\mathrm{d} \bar{W} \stackrel{!}{=} \mathrm{d} W_{\square}
$$

where

$$
\mathrm{d} \bar{W}=\overline{\boldsymbol{\sigma}}:[\mathrm{d} \overline{\boldsymbol{u}} \otimes \nabla]=\overline{\boldsymbol{\sigma}}: \mathrm{d} \overline{\boldsymbol{\varepsilon}},
$$

and

$$
\begin{aligned}
\mathrm{d} W_{\square} & =a_{\square, \mathrm{c}}\left(\boldsymbol{u}_{\mathrm{c}} ; \mathrm{d} \boldsymbol{u}_{\mathrm{c}}\right)+a_{\square, 1}\left(u_{\mathrm{s}, \mathrm{l}}, u_{\mathrm{s}, \perp} ; \mathrm{d} u_{\mathrm{s}, 1}\right)+a_{\square, \mathrm{b}}\left(u_{\mathrm{s}, \mathrm{l}}, u_{\mathrm{s}, \perp} ; \mathrm{d} u_{\mathrm{s}, \perp}\right) \\
& +c_{\square}\left(\lambda ; \mathrm{d} u_{\mathrm{s}, \perp}-\boldsymbol{e}_{\perp} \cdot \mathrm{d} \boldsymbol{u}_{\mathrm{c}}\right)+b_{\square}\left(u_{\mathrm{s}, 1}-\boldsymbol{e}_{\mathrm{l}} \cdot \boldsymbol{u}_{\mathrm{c}} ; \mathrm{d} u_{\mathrm{s}, 1}-\boldsymbol{e}_{l} \cdot \mathrm{d} \boldsymbol{u}_{\mathrm{c}}\right),
\end{aligned}
$$

are the macroscale virtual work (53) and the resolved virtual work on the subscale (38), respectively. In (A.12), we note that the term $c_{\square}\left(\mathrm{d} \lambda ; u_{\mathrm{s}, \perp}-\boldsymbol{e}_{\perp} \cdot \boldsymbol{u}_{\mathrm{c}}\right)$ has been removed, since the constraint is satisfied at the given solution. With help of the VMS ansatz, we have

$$
\begin{aligned}
\mathrm{d} W_{\square} & =a_{\square, \mathrm{c}}\left(\bullet ; \mathrm{d} \boldsymbol{u}_{\mathrm{c}}^{M}\right)+a_{\square, 1}\left(\bullet ; \mathrm{d} u_{\mathrm{s}, 1}^{M}\right)+a_{\square, \mathrm{b}}\left(\bullet ; \mathrm{d} u_{\mathrm{s}, \perp}^{M}\right)+b_{\square}\left(\bullet ; \mathrm{d} u_{\mathrm{s}, 1}^{M}-\boldsymbol{e}_{l} \cdot \mathrm{d} \boldsymbol{u}_{\mathrm{c}}^{M}\right) \\
& +c_{\square}\left(\bullet ; \mathrm{d} u_{\mathrm{s}, \perp}^{M}-\boldsymbol{e}_{\perp} \cdot \mathrm{d} \boldsymbol{u}_{\mathrm{c}}^{M}\right)+a_{\square, \mathrm{c}}\left(\bullet ; \mathrm{d} \boldsymbol{u}_{\mathrm{c}}^{s}\right)-b_{\square}\left(\bullet ; \boldsymbol{e}_{l} \cdot \mathrm{d} \boldsymbol{u}_{\mathrm{c}}^{s}\right)-c_{\square}\left(\bullet ; \boldsymbol{e}_{\perp} \cdot \mathrm{d} \boldsymbol{u}_{\mathrm{c}}^{s}\right) \\
& +a_{\square, 1}\left(\bullet ; \mathrm{d} u_{\mathrm{s}, 1}^{s}\right)+b_{\square}\left(\bullet ; \mathrm{d} u_{\mathrm{s}, 1}^{s}\right)+a_{\square, \mathrm{b}}\left(\bullet ; \mathrm{d} u_{\mathrm{s}, \perp}^{s}\right)+c_{\square}\left(\bullet ; \mathrm{d} u_{\mathrm{s}, \perp}^{s}\right),
\end{aligned}
$$


where the suppressed arguments refer to the current solution $\left(\boldsymbol{u}_{\mathrm{c}}, u_{\mathrm{s}, 1}, u_{\mathrm{s}, \perp}\right.$, and $\left.\lambda\right)$. Based on the expansion in (A.7)-(A.9), we identify that the arguments pertaining to the macroscale slip and penetration vanish, since

$$
\mathrm{d} u_{\mathrm{s}, 1}^{M}-\boldsymbol{e}_{l} \cdot \mathrm{d} \boldsymbol{u}_{\mathrm{c}}^{M}=\mathrm{d} u_{\mathrm{s}, \perp}^{M}-e_{\perp} \cdot \mathrm{d} \boldsymbol{u}_{\mathrm{c}}^{M}=0
$$

Furthermore, based on the definition of the forms in (39)-(43), we obtain

$$
a_{\square, \mathrm{c}}\left(\bullet ; \mathrm{d} \boldsymbol{u}_{\mathrm{c}}^{M}\right)+a_{\square, 1}\left(\bullet ; \mathrm{d} u_{\mathrm{s}, \mathrm{l}}^{M}\right)+a_{\square, \mathrm{b}}\left(\bullet ; \mathrm{d} u_{\mathrm{s}, \perp}^{M}\right)=\overline{\boldsymbol{\sigma}}: \mathrm{d} \bar{\varepsilon},
$$

where we recall the definition of $\overline{\boldsymbol{\sigma}}$ from (54). Using the relations (A.14) and (A.15), together with the canonical form in (A.1) - (A.5), we then obtain

$$
\mathrm{d} W_{\square}=\overline{\boldsymbol{\sigma}}: \mathrm{d} \overline{\boldsymbol{\varepsilon}}+d_{\square}\left(\hat{\boldsymbol{\sigma}}_{\mathrm{c}} ; \mathrm{d} \boldsymbol{u}_{\mathrm{c}}^{s}\right) .
$$

Finally, the variation of (A.5) for a change d $\bar{\varepsilon}$ gives

$$
d_{\square}\left(\delta \hat{\boldsymbol{\sigma}}_{\mathrm{c}} ; \mathrm{d} \boldsymbol{u}_{\mathrm{c}}^{\mathrm{s}}\right)=0 \quad \forall \delta \hat{\boldsymbol{\sigma}}_{\mathrm{c}} \in \mathbb{R}^{2 \times 2} .
$$

Hence, we conclude that the second term in (A.16) vanishes, and thus

$$
\mathrm{d} W_{\square}=\overline{\boldsymbol{\sigma}}: \mathrm{d} \bar{\varepsilon}=\mathrm{d} \bar{W} .
$$

as stated in (A.11).

\section{References}

[1] fib. Bond of reinforcement in concrete: state-of-the-art report. Bulletin 10 (fib Fédération internationale du béton), International Federation for Structural Concrete, 2000.

[2] Daoud A, Maurel O, Laborderie C. 2D mesoscopic modelling of bar-concrete bond. Engineering Structures 2013; 49:696-706.

[3] ACI Committee 408. ACI 408.2R Report on bond of steel reinforcing bars under cyclic loads. Technical Report, American Concrete Institute 2012.

[4] fib. Model code for concrete structures 2010. Wiley-VCH Verlag GmbH \& Co. KGaA, 2013.

[5] Domínguez N, Fernández MA, Ibrahimbegovic A. Enhanced solid element for modelling of reinforced concrete structures with bond-slip. Computers and Concrete 2010; 7(4):347-364.

[6] Kouznetsova V, Brekelmans WAM, Baaijens FPT. An approach to micro-macro modeling of heterogeneous materials. Computational Mechanics 2001; 27(1):37-48.

[7] Kouznetsova V, Geers MGD, Brekelmans WAM. Multi-scale constitutive modelling of heterogeneous materials with a gradient-enhanced computational homogenization scheme. International Journal for Numerical Methods in Engineering 2002; 54(8):1235-1260.

[8] Wriggers P, Moftah SO. Mesoscale models for concrete: Homogenisation and damage behaviour. Finite Elements in Analysis and Design 2006; 42(7):623-636.

[9] Pichler C, Lackner R, Mang HA. A multiscale micromechanics model for the autogenousshrinkage deformation of early-age cement-based materials. Engineering Fracture Mechanics 2007; 74(1-2):34-58. 
[10] Wang Z, Kwan A, Chan H. Mesoscopic study of concrete I: generation of random aggregate structure and finite element mesh. Computers 85 Structures 1999; 70(5):533-544.

[11] Unger JF, Eckardt S. Multiscale modeling of concrete. Archives of Computational Methods in Engineering 2011; 18(3):341-393.

[12] Nilenius F, Larsson F, Lundgren K, Runesson K. Macroscopic diffusivity in concrete determined by computational homogenization. International Journal for Numerical and Analytical Methods in Geomechanics 2013; 37(11):1535-1551.

[13] Nguyen VP, Lloberas-Valls O, Stroeven M, Sluys LJ. Homogenization-based multiscale crack modelling: From micro-diffusive damage to macro-cracks. Computer Methods in Applied Mechanics and Engineering 2011; 200(9-12):1220-1236.

[14] Coenen EWC, Kouznetsova VG, Bosco E, Geers MGD. A multi-scale approach to bridge microscale damage and macroscale failure: A nested computational homogenization-localization framework. International Journal of Fracture 2012; 178(1-2):157-178.

[15] Karamnejad A, Nguyen VP, Sluys LJ. A multi-scale rate dependent crack model for quasi-brittle heterogeneous materials. Engineering Fracture Mechanics 2013; 104:96-113.

[16] Le JL, DesHarnais M, Xue B, Pang SD, Du H. A two-scale computational model for thermomechanical analysis of reinforced concrete frames. Engineering Structures 2015; 105:137-151.

[17] Sun B, Li Z. Adaptive concurrent multi-scale FEM for trans-scale damage evolution in heterogeneous concrete. Computational Materials Science 2015; 99:262-273.

[18] Sun B, Wang X, Li Z. Meso-scale image-based modeling of reinforced concrete and adaptive multi-scale analyses on damage evolution in concrete structures. Computational Materials Science 2015; 110:39-53.

[19] Sun B, Li Z. Multi-scale modeling and trans-level simulation from material meso-damage to structural failure of reinforced concrete frame structures under seismic loading. Journal of Computational Science 2016; 12:38-50.

[20] Larsson F, Runesson K, Su F. Variationally consistent computational homogenization of transient heat flow. International Journal for Numerical Methods in Engineering 2010; 81(13):16591686.

[21] Öhman M, Runesson K, Larsson F. On the variationally consistent computational homogenization of elasticity in the incompressible limit. Advanced Modeling and Simulation in Engineering Sciences 2015; 2(1):1-29.

[22] Larsson F, Runesson K. RVE computations with error control and adaptivity: the power of duality. Computational Mechanics 2007; 39(5):647-661.

[23] Patzák B. OOFEM - an object-oriented simulation tool for advanced modeling of materials and structures. Acta Polytechnica 2012; 52(6):59-66.

[24] CEN. Eurocode 2: Design of concrete structures. Part 1: General rules and rules for buildings. European Committee for Standardization 2004; .

[25] Mazars J. A description of micro- and macroscale damage of concrete structures. Engineering Fracture Mechanics 1986; 25(5):729-737. 
[26] Mazars J, Pijaudier-Cabot G. Continuum damage theory - application to concrete. Journal of Engineering Mechanics 1989; 115(2):345-365.

[27] Oliver J, Cervera M, Oller S, Lubliner J. Isotropic damage models and smeared crack analysis of concrete. Computer Aided Analysis and Design of Concrete Structures, 1990; 945-957.

[28] Svenning E, Fagerström M, Larsson F. Computational homogenization of microfractured continua using weakly periodic boundary conditions. Computer Methods in Applied Mechanics and Engineering 2016; 299:1-21.

[29] Coenen EWC, Kouznetsova VG, Geers MGD. Novel boundary conditions for strain localization analyses in microstructural volume elements. International Journal for Numerical Methods in Engineering 2012; 90(1):1-21.

[30] Balay S, Abhyankar S, Adams M, Brown J, Brune P, Buschelman K, Dalcin L, Eijkhout V, Gropp W, Kaushik D, et al. PETSc Web page 2017. URL http://www.mcs.anl.gov/petsc.

[31] Hill R. Elastic properties of reinforced solids: Some theoretical principles. Journal of the Mechanics and Physics of Solids 1963; 11(5):357-372. 